\title{
Study of pulsed neon xenon VUV radiating low pressure plasmas for mercury free fluorescent sign optimization
}

\author{
E Robert, S Point, S Dozias, R Viladrosa and J M Pouvesle \\ GREMI, CNRS-Polytech' Orléans, 14 rue d'Issoudun, BP 6744, 45067 Orléans Cedex 2, France \\ E-mail : eric.robert@univ-orleans.fr
}

\begin{abstract}
This work deals with the study and optimization of mercury free fluorescent discharge tubes for publicity lightning applications. The experimental set up allows for the time resolved spectroscopy from $110 \mathrm{~nm}$ up to $900 \mathrm{~nm}$, the photometric characterization in a large volume integrating sphere, and the current and voltage measurement of microsecond duration signals delivered by lab-developed pulsed drivers. The glow and afterglow radiative process analysis indicates that the best performance measured with the pulsed excitation of rare gas plasma, in comparison with the conventional AC excitation, essentially originates from the efficient plasma relaxation during the afterglow at the benefit of the VUV resonance line radiated at $146.9 \mathrm{~nm}$ for xenon. The fit of the VUV time resolved experimental measurements with the results issued from a simplified kinetic model of neon xenon plasmas, evidence the crucial role of molecular ions production during the glow phase and of their radiative recombination during the afterglow. The pulse duration and the gas mixture pressure appear as two experimental parameters which influence, studied over an extended range, has been demonstrated to allow a significant sign performance enhancement. There exists an optimum pulse duration range, which results in the appearance of limited stepwise excitation and ionization processes, favourable for an intense afterglow VUV production. The pressure dependence study shows that the best performance for pulsed excitation are obtained in Ne/Xe (100/1) mixtures around $50 \mathrm{mbar}$, at the difference of $\mathrm{AC}$ driven $\mathrm{Ne} / \mathrm{Xe}$ plasma for which the best conditions were reported to be of a few mbar. This pressure increase results both in the VUV and sign light output enhancement and the successful continuous operation of pulsed mercury free signs for time as long as 4000 hours with neither electrode erosion, nor glass or phosphor degradation nor chromatic coordinate variation. For the green phosphor covered, $65 \mathrm{~cm}$ long and $13 \mathrm{~mm}$ inside diameter signs, the efficiency of pulsed neon xenon discharge likely to be operated for a few thousand hours reaches $50 \%$ of that of the same tube filled with mercury based mixtures.
\end{abstract}

PACS:

\section{Introduction}

As enlighten through the last issues of the international lighting conference [1-3], there exists a growing interest and research effort devoted to the development of new environmentally friendly substitute to the mercury based fluorescent technology. Dealing with gas discharge technology, various 
excitation techniques of gas mixture plasmas are studied or already used in different fields of applications. DC and AC discharges using hot filaments or cold cathodes, pulsed excitation of such discharges, but also RF excitation of electrode-less lamps have for example been studied for a long time for lightning applications. The main issue is to achieve an efficient excitation of the phosphor luminescence by an intense UV-VUV emitter. Rare gas mixtures, including xenon, close to the atmospheric pressure have already been applied for display applications. Major progress have been reported in the optimization [4-7], and modelling [7-9], of low pressure rare gas mixtures based on $\mathrm{Xe}$ resonance line. A large effort is still devoted to the study of the three following and sometime antagonist objectives: light output flux, lamp efficiency and the lamp lifetime.

At GREMI, such a research was partly supported by the AUPEM SEFLI company for years, to find out $\mathrm{Hg}$ free alternatives to the luminous signs used for publicity lightning. Signs represent a specific case for the search of the efficient and long living operation of a plasma produced by a high voltage power supply connected across cold hollowed electrodes. While important, the luminous efficiency of signs is not such a crucial parameter in comparison with that of general lighting lamps, but very stringent conditions need to be fulfilled for publicity lighting devices such as a few tens of thousand hour lifetime and a very limited chromatic coordinate variation during the whole sign operation. Preliminary works $[10,11]$ have shown evidence for the potentialities of pulsed excited low pressure rare gas mixtures which were shown to allow for a three time enhancement of the luminous output and energy efficiency in comparison with conventional AC excitation.

In this work, the time resolved spectroscopic study of neon-xenon plasma excited by microsecond and sub microsecond duration pulses delivered at repetition rate ranging from a few hundreds of $\mathrm{Hz}$ up to a few tens of $\mathrm{kHz}$, the photometric and ageing characterization of sealed signs is proposed. The section 2 describes the experimental set up used for fluorescent tube conditioning, the pulsed driver technology, the electrical measurements, the VUV to near infrared spectroscopic study and the photometric measurements. The detailed analysis of the plasma emission during the glow phase and the afterglow documented in the section 3, allow for an approach of the main kinetics pathways through a simplified model of the molecular ion formation and of their successive recombination leading to a very significant population of the xenon resonance level also described in this section. The experimental perspectives inferred from the modelling have been investigated both for the pulse duration and gas mixture pressure as depicted in section 4. The section 5 deals with the lifetime and efficiency measurements and the last section draws some conclusion and suggests a few perspectives to this work.

\section{Pulsed fluorescent sign experimental set- up}

The experimental set-up used for the fluorescence tube conditioning, ignition and diagnostics is presented in figure 1 . The borosilicate discharge reactor is manufactured by the AUPEM SEFLI partner and consists in a T-shaped fluorescent tube, equipped with two conventional hollow electrodes. The electrodes are iron/nickel cylinders $40 \mathrm{~mm}$ in length and $10 \mathrm{~mm}$ in diameter, the inside of these electrodes is covered with an electron emitter barium fluoride layer. The $3 \mathrm{~mm}$ thick ceramic ring at their extremity is used to prevent from strong sputtering at the plasma facing electrode edge. Tubes with three internal diameters $(10,13$ and $18 \mathrm{~mm})$ and with length, defined as the distance between the two electrode ceramic rings, ranging from $10 \mathrm{~cm}$ up to $1 \mathrm{~m}$, have been used in this work. When no specific indication is specified, the results presented through this manuscript refer to $13 \mathrm{~mm}$ inside diameter and $50 \mathrm{~cm}$ inter electrodes long tubes. The inner surface of the tube is covered with a zinc silicate manganese activated phosphor prepared and deposited by the AUPEM SEFLI company following the same procedure as that used for mercury based conventional signs. This green phosphor presents a peak emission located around 525 $\mathrm{nm}$. The $\mathrm{T}$ branch of the fluorescent tube is first connected to the gas handling system, including gas flow controllers, pressure gauges, a needle valve and a vacuum pump to monitor the gas mixture composition. Gas flow rates, ranging from 0,1 to $50 \mathrm{~cm}^{3} \mathrm{~s}^{-1}$, are used during experiments to allow for a parametric 
study of the gas mixture composition influence on the tube performance without requiring the realization of a considerable number of sealed signs. Previous measurements $[6,10]$ have shown that this fluorescent tube experimental procedure is valid for the prediction of the behaviour of sealed signs. Before the filling with high purity gases, N 45 or higher grade supplied by Air Liquide company, the tube is heated up to about $350{ }^{\circ} \mathrm{C}$, via the neon tube bombarder, this temperature being measured on the outer surface of the glass at the centre of the tube. This procedure allows the removal of the impurities trapped in the glass, the phosphor and the metallic electrodes of the sign through the vacuum assembly of the bombarder.

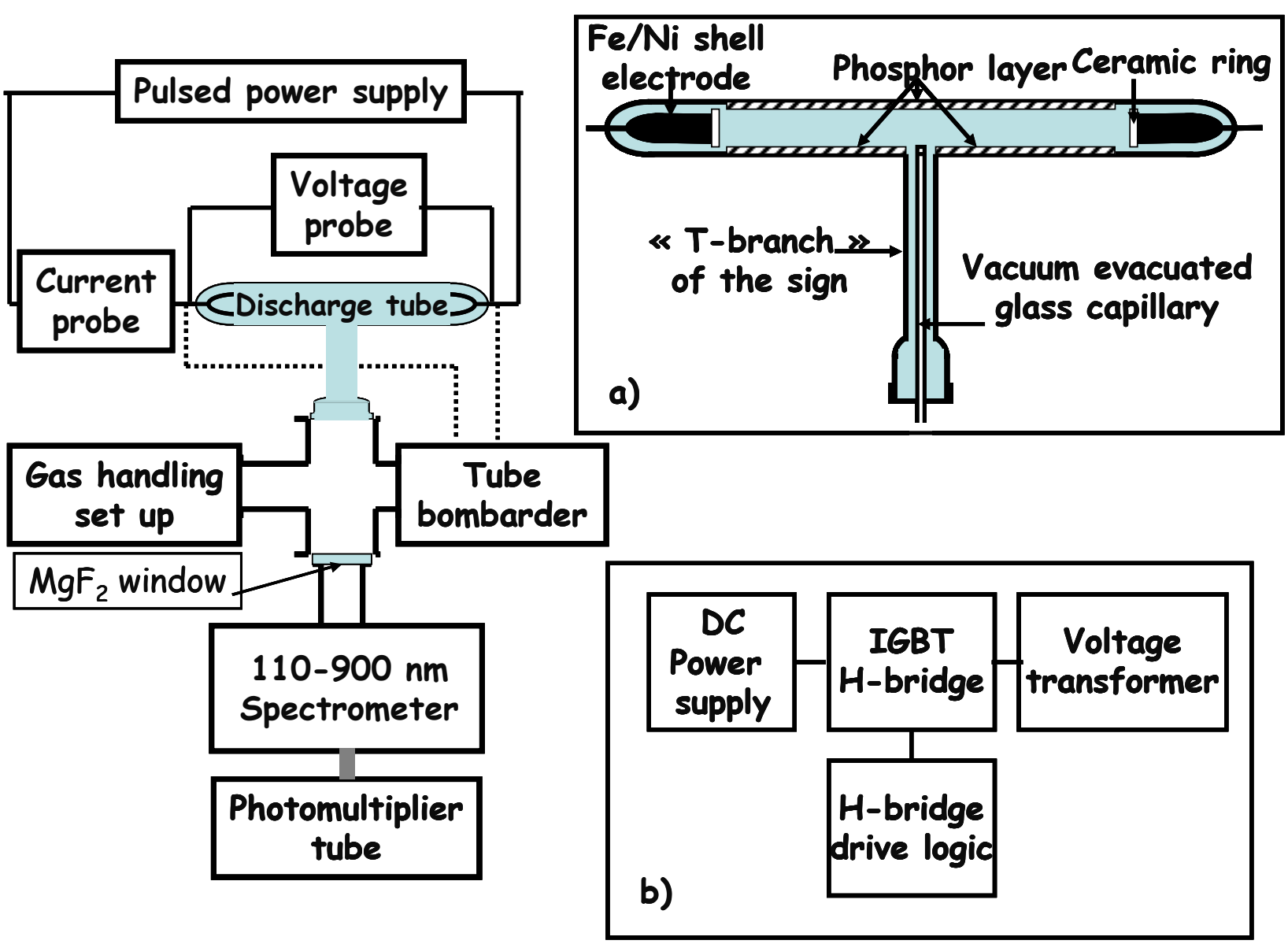

Figure 1. Whole experimental set-up on the left, inset a) provides some details on the fluorescent tube configuration, inset b) is a schematic of the pulsed power supply.

Finally, the $\mathrm{T}$ branch is optically connected to a VUV-UV-visible (110-900 nm) 0.4 focal length Acton Research spectrometer equipped with two gratings (300 and 1200 grooves/mm respectively). Photomultiplier tubes (Hamamatsu R1220 and R955) are connected to the spectrometer for the measurement of the time resolved emission spectra of the discharge. A PImax ICCD camera, not shown in figure 1, was also used for imaging experiments discussed in section 4. The VUV measurement of the xenon $146.9 \mathrm{~nm}$ resonance line radiation has been performed through a vacuum evacuated glass capillary with an inside diameter of $3 \mathrm{~mm}$, equipped with a $\mathrm{MgF}_{2}$ window. This VUV window is set at the same position than the phosphor layer plane, through the $\mathrm{T}$ branch. In this way, the radiation trapping in the xenon gas is included in the measurement and corresponds to the phosphor excitation condition.

The inset b) in figure 1 gives some schematic description of the pulsed power supply developed in this work. The pulsed power 
supply articulates around three main subassemblies. A DC power supply is used as a voltage source which amplitude ranges from about $100 \mathrm{~V}$ up to $1250 \mathrm{~V}$ and is typically set between 300 and $400 \mathrm{~V}$. An IGBT based Hbridge together with its driving logic is used to produce voltage pulses with adjustable duration, in the microsecond range, and adjustable repetition rate, in the kilohertz range. The full $\mathrm{H}$-bridge set up produces voltage cycles having one positive and one negative polarity pulse, which is required for fluorescent tube operation to avoid cataphoresis effect and to allow a similar degradation of both tube electrodes. These voltage pulses are finally applied to the primary coil of a high voltage transformer having a magnification factor of about 5, the secondary coil is connected across the fluorescent tube which requires voltage amplitude around one kilovolt to ignite. As discussed in section 4 , some experiments have been performed with charging the H-bridge up to $1250 \mathrm{~V}$ and without using the voltage transformer to suppress power dissipation through this element and to obtain faster voltage rising edges and consequently shorter duration pulses. Voltage and respectively current measurements were performed using Elditest Electronic GE $3421 \quad(100 \mathrm{MHz}$ bandwidth) and Tektronix TCP202 (50 MHz bandwidth) probes respectively connected to a Tektronix 510 oscilloscope.

Finally, photometric characterization consisted in the measurement of illuminance through a Minolta chroma meter, model xy-DF, and the measurement of the output visible flux through a $1 \mathrm{~m}$ diameter SLM-40 SphereOptics integrating sphere.

\section{Pulsed neon xenon plasma kinetics}

This section describes the essential features of the glow phase of neon xenon plasma produced with the use of the pulsed driver described in the previous section. The figure 2 presents the waveforms of the voltage and current signals together with those of the xenon resonant line radiated at $146.9 \mathrm{~nm}$, the xenon infrared line at $823.1 \mathrm{~nm}$, one of the most intense visible neon lines emitted at 640.2 $\mathrm{nm}$, and the strongest ionic xenon line measured at $529.2 \mathrm{~nm}$. This recording was obtained for a 45 mbar mixture containing $1 \%$ of xenon and excited by five microsecond duration pulses delivered at a repetition rate of two kilohertz.
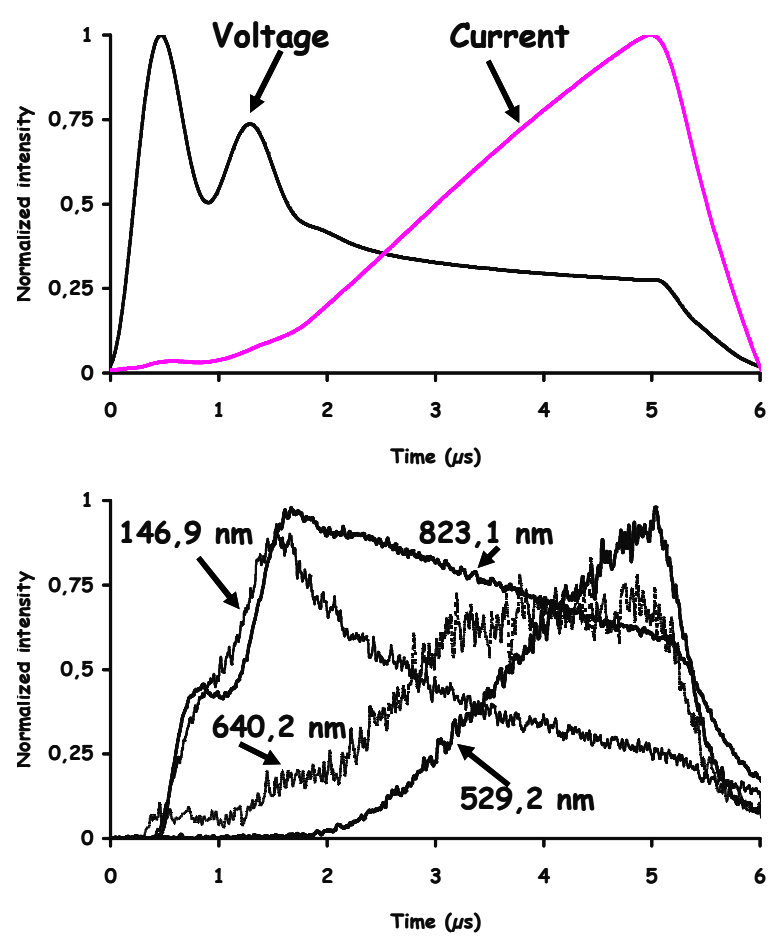

Figure 2. Temporal evolution during the glow phase of: voltage (dark trace) and current (grey trace) waveforms upper graph, and on lower graph of : the xenon resonant level at $146.9 \mathrm{~nm}$, the xenon infrared line at $823.1 \mathrm{~nm}$, the xenon ion radiation at $529.2 \mathrm{~nm}$ and the neon fluorescence at $640.2 \mathrm{~nm}$.

The glow is described by splitting this phase in two different stages. In the experimental conditions of figure 2 , the transition between the two glow phase stages is measured two microsecond after the onset of the current pulse occurring at the time origin $(\mathrm{t}=0)$. At the instant of $2 \mu \mathrm{s}$, the current waveform turns from a low amplitude and slowly increasing signal to a quite linear ramp. The peak amplitude at the end of the current ramp, i.e. at the end of the current pulse, is of a few A. This transition corresponds also with the simultaneous decay of the resonance line emission, the strong enhancement of the neon line intensity and the rise of ionic xenon line radiation. The transition instant is essentially dependent on the pulse repetition rate, it occurs earlier for higher repetition rate and later for lower repetition rate.

The consideration of electron-impact excitation cross sections for neon and xenon both from the ground state and their metastable 
levels [12] together with the evolution of the amplitude of the current allow for a schematic description of the main kinetic reactions involved in the plasma species temporal evolution and the evolution in the electronic temperature during the pulse excitation. At the pulse onset, the plasma from the previous pulse is largely recombined, the tube impedance is high, in the $\mathrm{kOhm}$ range, the electronic density is low and the electronic temperature is high which is favourable for the direct excitation of the xenon and neon excited levels from their respective ground states. This allows for the population of the two rare gas metastable levels, the efficient excitation of the xenon $146.9 \mathrm{~nm}$ resonance line, and neon $73.6 \mathrm{~nm}$ transition, not measured in this work, but at the origin of the phosphor excitation in pure neon plasma [10]. These excitation processes require the participation of electron having energies above the threshold of $8.3 \mathrm{eV}$ and respectively $16.62 \mathrm{eV}$ for xenon and respectively neon. Then as the current density increases, the positive column plasma is established, the typical electronic temperature is around $1 \mathrm{eV}$, and the main electron impact reactions involve electrons having energies of a few $\mathrm{eV}$. These plasma parameters are very favourable for stepwise excitation and ionization in the case of xenon and stepwise excitation for neon [12]. The spectroscopic analysis reveals indeed the decrease of all the observed xenon transitions and the large increase of all the neon transitions during the second stage of the glow phase. Stepwise excitation processes from xenon lower excited levels towards higher energy levels is depicted in figure 2 through the much more pronounced decrease of the resonant transition in comparison with that of the infrared line during the second phase of the glow signal. The production of xenon ions from xenon excited levels, the population of neon excited levels and the consecutive increasing importance of penning reaction lead to a strong rise of the electron density. This also leads, with a small time delay of less than 0.5 microsecond, to the production of high energy lying singly charged excited levels of xenon as depicted in figure 2 through the 529.2 $\mathrm{nm}$ line waveform which upper level have an energy of about $26 \mathrm{eV}$. The excitation of neutral neon and singly charged xenon transitions lasts up to the end of the current pulse.
The schematic description of the plasma kinetics during the glow phase indicates than the main portion of the power input lead to the production of visible radiation which are of no use for the phosphor excitation. When the pulse repetition rate is increased while the pulse duration is shortened to keep a constant electrical input power, the plasma recombination and relaxation is interrupted resulting in higher metastable state and electronic densities at the time of application of the next voltage pulse. This induces a faster transition between the two glow phases and this effect is even much more important if the pulse duration is not reduced, i.e. if the electrical power is increased. The neon xenon pulsed plasma kinetics analysis also confirms the unlikely stepwise excitation processes occurring with larger and larger amplitude when using AC excitation. With AC excitation, the increase of the power input across the fluorescent tube above about $5 \mathrm{~W}$ unfortunately led to the saturation at a constant level of the phosphor fluorescence which does not allow for the production of illuminance levels of interest for signs and lightning applications [10].

In the case of pulsed excitation, the next section evidences how the plasma recombination during the afterglow phase can be optimized to achieve a significant recovery of the power spent for the production of non $\mathrm{UV}$ radiating species. The figure 3 presents the temporal waveforms, of the resonant xenon transition at $146.9 \mathrm{~nm}$, the $823.1 \mathrm{~nm}$ transition and of the most intense xenon visible radiation at $467.1 \mathrm{~nm}$. The three signals are normalized to their respective maximum during the glow phase which lasts during the first ten microseconds. The fluorescence during the afterglow is measured up to the application of the next voltage pulse and is presented in figure 3 over 150 microseconds. The afterglow signal for visible neon transitions was measured to be negligible in our experimental conditions. Two essential features appear from figure 3. First the integration of the signal over the whole afterglow duration leads to the conclusion that the larger part of VUV radiation is emitted during the afterglow in comparison with the emission measured during the glow phase. Second, the radiative cascades and quenching reactions induce that the larger afterglow contribution is obtained for the lower lying energy level, as revealed by the 
comparison of the $146.9 \mathrm{~nm}$ signal with other xenon transitions, which is a very favourable situation for the production of VUV radiation.

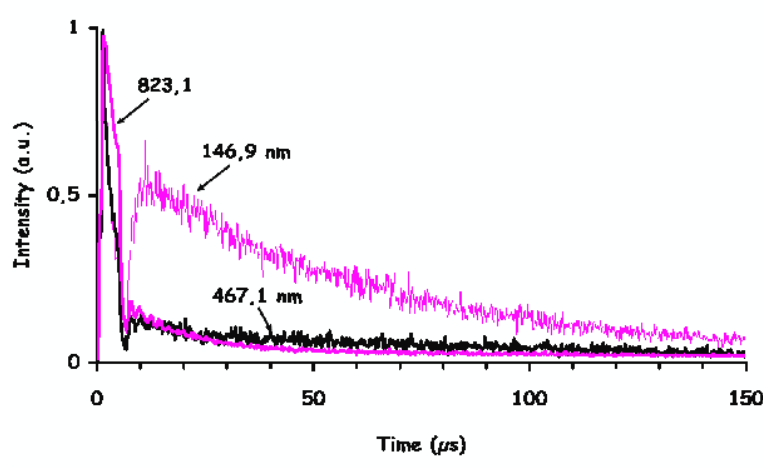

Figure 3. Temporal evolutions of the xenon resonant radiation at $146.9 \mathrm{~nm}$, the xenon infrared line at $823.1 \mathrm{~nm}$, and of the xenon visible emission at $467.1 \mathrm{~nm}$ (dark trace). The three waveforms are normalized to their respective maximum during the glow phase. Ne/Xe (100/1) mixture at a pressure of 45 mbar was used.

The development of a simplified kinetic model likely to allow the interpretation of the fluorescence signals measured during the afterglow phase for a radialy uniform positive column section was performed including the reactions summarized in table 1 together with the initial conditions for the different species densities. This initial densities are representative of a typical low pressure glow discharge and the different values were fitted to obtain the best matching between the calculated $\mathrm{Xe}^{*}$ density and the $146.9 \mathrm{~nm}$ waveform. Besides xenon and neon ground state atoms, six species have been included in the modelling: electrons, xenon ions, neon and xenon excited atoms which are both considered in a rough approach as two unique states, the xenon dimer ion and the heteronuclear neon xenon ions. As described in section 4.2, the termolecular reactions leading to the production of xenon dimer, $\mathrm{Xe}_{2}{ }^{*}$, were not considered in the modelling as no evidence for the population of excited dimer was spectroscopically observed. On the contrary, the corresponding termolecular reactions involving the xenon ion and leading to the production of $\mathrm{Xe}_{2}^{+}$or $\mathrm{NeXe}^{+}$molecular ions present reaction rate one order of magnitude higher than those for $\mathrm{Xe}^{*}$ atoms and the xenon ion density is also much than one order of magnitude higher than the excited xenon state density. Our main goal was to describe the main kinetic paths leading to the strong afterglow signal depicted in figure 3 . The initial value of the $\mathrm{Xe}^{*}$ species, set to $1.6 \mathrm{x}$ $10^{10} \mathrm{~cm}^{-3}$ in the modelling, appears in relatively good agreement with that of [7] in which respective axis densities of $8 \times 10^{11}, 2 \mathrm{x}$ $10^{11}, 2 \times 10^{10}$, and $5 \times 10^{9}$ were determined for $\mathrm{Xe}^{*}\left(1 \mathrm{~s}_{5}, 1 \mathrm{~s}_{4}, 1 \mathrm{~s}_{3}\right.$ and $\left.1 \mathrm{~s}_{2}\right)$ levels. In the present work, no radial analysis of the plasma column was considered and only one neutral excited xenon state was introduced. It was shown in [7], that at the wall species densities could be at least ten times smaller that the corresponding on axis values. Once again, the detailed modelling on the plasma was not the goal of the present paper, which would require a deeper analysis of the radial density profiles, the electron temperature calculation, the insertion of various xenon and neon states and corresponding reaction path and rates. The initial electron density, set to $6 \times 10^{11} \mathrm{~cm}^{-3}$ in the modelling, is larger than that of $4 \times 10^{11}$ $\mathrm{cm}^{-3}$ obtained in [7], while being of the same order of magnitude. This higher electron density value has to be moderated by the consideration of the pulsed, low duty cycle excitation mode of the plasma in our study. Typical duty cycle of $1 \%$, corresponding to microsecond pulses delivered at $\mathrm{kHz}$ repetition rates, result in a mean electron density of a few $10^{10} \mathrm{~cm}^{-3}$ while in [7] the excitation consisted in a AC $60 \mathrm{~mA}$ waveform. The neon excited level density of $10^{8} \mathrm{~cm}^{-3}$ is consistent with the consideration that even with small xenon admixtures, the density of excited neon atoms remain far below those of xenon excited levels. Finally, the initial electron temperature for the afterglow phase description, set to $0.7 \mathrm{eV}$ in the modelling, is consistent with the observed xenon and neon line intensities behaviour previously described in figure 3 . The temporal evolution of the electron temperature, that is the fast decreasing during the first fifty microseconds during the afterglow, was shown to be crucial in the fit of the experimental records. It could be pointed out that such rapid thermalization of electron population was previously documented in [17] where the large xenon density growth at the onset of the afterglow was connected with a two times reduction of the electron temperature within a $20 \mu$ s duration, this evolution being very close to that inferred in our study to achieve the best fit between calculation and experimental data. 
The figure 4 presents the comparison of the $147 \mathrm{~nm}$ fluorescence signal, measured for a $\mathrm{Ne} / \mathrm{Xe}(100 / 1)$ mixture at a pressure of 45 mbar excited by two microsecond duration pulses, with the results of the modelling including the full set of reactions and initial conditions presented in table 1 (black trace) and without the consideration of R9 and R10 (thin grey trace).

The calculations indicate that the main process responsible for the plasma recombination is the dissociative recombination of molecular ions $\left(\mathrm{Xe}_{2}{ }^{+}\right.$and $\left.\mathrm{NeXe}^{+}\right)$while in our experimental conditions the collisionnal radiative reaction R11 plays no significant role in the plasma relaxation. This latter process would lead to an afterglow signal which would peak with a delay larger than 400 microseconds which is not measured in the experiments. It appears that the recombination of our pulsed neon xenon plasma, produced with xenon partial pressure of about $0.5 \mathrm{mbar}$, occurs at electron temperature lower than $0.7 \mathrm{eV}$, electron density of a few $10^{11} \mathrm{~cm}^{-3}$, and essentially involves dissociative recombination reactions. This regime appears in between the previously reported and modelled xenon low pressure (0.07 mbar), high density (a few $10^{14} \mathrm{~cm}^{-3}$ ), low electron temperature (below $1 \mathrm{eV}$ ) and the constricted high xenon pressure (13 mbar), low electron density $\left(10^{11} \mathrm{~cm}^{-3}\right)$ and high electron temperature $(3.5 \mathrm{eV})$ modes of operation of pulsed low voltage discharges [17]. The model also confirms the fast decrease of the electronic temperature after the current pulse end, as the best fit were obtained if the temperature falls from $0,7 \mathrm{eV}$ at the end of the pulse down to a few tenths of $\mathrm{eV}$ in the first tens of microseconds during the afterglow. Finally, the rough modelling of the afterglow phase has been used to determine the most promising pathways for the optimization of VUV production in neon xenon pulsed driven discharges. The table 1 indeed indicates that $\mathrm{R} 9, \mathrm{R} 10$ and the formation reactions R4, R5 have a strong influence on the production of VUV radiation so that it appears worthwhile to study higher pressure and higher density plasma.

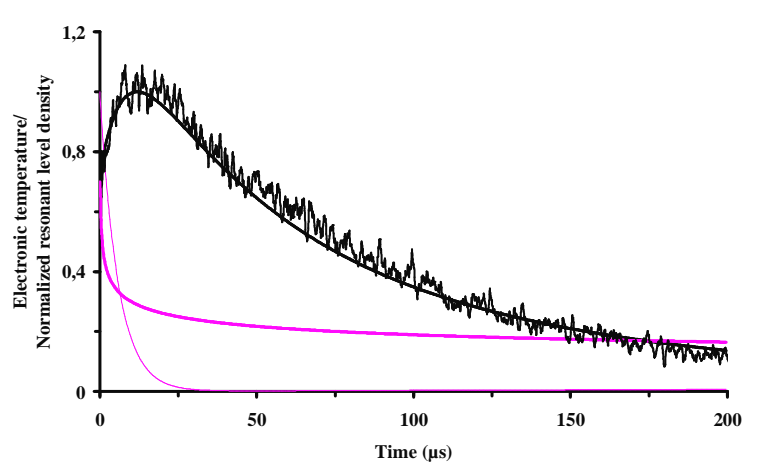

Figure 4. Comparison of the fluorescence signal at $146.9 \mathrm{~nm}$ with the result of the modelling including the full reaction set of table 1 (black smooth trace) and without R9 and R10 (thin grey trace). The thick grey trace presents the temporal evolution of the electronic temperature selected in the modelling.

Table 1. Selected reactions, rate coefficients and species initial densities at the beginning of the afterglow.

\begin{tabular}{|c|c|c|c|c|c|c|c|}
\hline \multicolumn{4}{|l|}{ Reaction } & Label & \multicolumn{2}{|c|}{ Rate coefficient } & Reference \\
\hline \multicolumn{2}{|l|}{$\mathrm{Xe}+\mathrm{Ne}^{*}$} & $\rightarrow$ & $\mathrm{Xe}^{+}+\mathrm{Ne}+\mathrm{e}^{-}$ & R1 & \multicolumn{2}{|c|}{$7,5 \times 10^{-11} \mathrm{~cm}^{3} \mathrm{~s}^{-1}$} & [13] \\
\hline \multicolumn{2}{|l|}{$\mathrm{Xe}+\mathrm{Ne}^{*}$} & $\rightarrow$ & $\mathrm{NeXe}^{+}+\mathrm{e}^{-}$ & $\mathrm{R} 2$ & \multicolumn{2}{|c|}{$2,3 \times 10^{-11} \mathrm{~cm}^{3} \mathrm{~s}^{-1}$} & {$[13]$} \\
\hline \multirow{2}{*}{\multicolumn{2}{|c|}{$\begin{array}{l}\mathrm{Xe}^{+}+2 \mathrm{Xe} \\
\mathrm{Xe}^{+}+\mathrm{Xe}+\mathrm{Ne}\end{array}$}} & $\rightarrow$ & $\mathrm{Xe}_{2}^{+}+\mathrm{Xe}$ & R3 & \multicolumn{2}{|c|}{$2,0 \times 10^{-31} \mathrm{~cm}^{6} \mathrm{~s}^{-1}$} & [14] \\
\hline & & $\rightarrow$ & $\mathrm{Xe}_{2}^{+}+\mathrm{Ne}$ & R4 & \multicolumn{2}{|c|}{$1,5 \times 10^{-31} \mathrm{~cm}^{6} \mathrm{~s}^{-1}$} & {$[15]$} \\
\hline \multicolumn{2}{|c|}{$\mathrm{Xe}^{+}+2 \mathrm{Ne}$} & $\rightarrow$ & $\mathrm{NeXe}^{+}+\mathrm{Ne}$ & R5 & \multicolumn{2}{|c|}{$1,5 \times 10^{-31} \mathrm{~cm}^{6} \mathrm{~s}^{-1}$} & [15] \\
\hline \multicolumn{2}{|c|}{$\mathrm{NeXe}^{+}+\mathrm{Xe}$} & $\rightarrow$ & $\mathrm{Xe}^{+}+\mathrm{Ne}+\mathrm{Xe}$ & R6 & \multicolumn{2}{|c|}{$5,0 \times 10^{-10} \mathrm{~cm}^{3} \mathrm{~s}^{-1}$} & [16] \\
\hline \multicolumn{2}{|c|}{$\mathrm{NeXe}^{+}+\mathrm{Xe}$} & $\rightarrow$ & $\mathrm{Xe}_{2}^{+}+\mathrm{Ne}$ & R7 & \multicolumn{2}{|c|}{$5,0 \times 10^{-12} \mathrm{~cm}^{3} \mathrm{~s}^{-1}$} & {$[16]$} \\
\hline \multicolumn{2}{|c|}{$\mathrm{Xe}_{2}^{+}+\mathrm{e}^{-}$} & $\rightarrow$ & $\mathrm{Xe}^{+}+\mathrm{Xe}+\mathrm{e}^{-}$ & R8 & \multicolumn{2}{|c|}{$1,0 \times 10^{-7} \mathrm{~cm}^{3} \mathrm{~s}^{-1}$} & [14] \\
\hline \multicolumn{2}{|l|}{$\mathrm{Xe}_{2}^{+}+\mathrm{e}^{-}$} & $\rightarrow$ & $\mathrm{Xe}^{*}+\mathrm{Xe}$ & R9 & \multicolumn{2}{|c|}{$2,3 \times 10^{-6} \times \mathrm{T}_{\mathrm{e}}^{-0,7} \mathrm{~cm}^{6} \mathrm{~s}^{-1}$} & {$[17]$} \\
\hline \multicolumn{2}{|c|}{$\mathrm{NeXe}^{+}+\mathrm{e}^{-}$} & $\rightarrow$ & $\mathrm{Xe}^{*}+\mathrm{Ne}$ & $\mathrm{R} 10$ & \multicolumn{2}{|c|}{$2,0 \times 10^{-7} \times \mathrm{T}_{\mathrm{e}}^{-0,5} \mathrm{~cm}^{6} \mathrm{~s}^{-1}$} & {$[18]$} \\
\hline \multicolumn{2}{|c|}{$\mathrm{Xe}^{+}+2 \mathrm{e}^{-}$} & $\rightarrow$ & $X e^{*}+e^{-}$ & R11 & \multicolumn{2}{|c|}{$2,0 \times 10^{-27} \times \mathrm{T}_{\mathrm{e}}^{-4,5} \mathrm{~cm}^{6} \mathrm{~s}^{-1}$} & [17] \\
\hline \multicolumn{2}{|l|}{$\mathrm{Xe}^{*}$} & $\rightarrow$ & $\mathrm{Xe}+\mathrm{hv}$ & $\mathrm{R} 12$ & \multicolumn{2}{|c|}{$10^{-5} \mathrm{~s}^{-1}$} & [17] \\
\hline $\mathrm{T}_{\mathrm{e}}$ & & $\mathrm{e}^{-}$ & $\mathrm{Xe}^{+}$ & $\mathrm{Ne}^{*}$ & $\mathrm{Xe}^{*}$ & $\mathrm{Xe}_{2}^{+}$ & $\mathrm{NeXe}^{+}$ \\
\hline $0,7 \mathrm{eV}$ & $6 x$ & $10^{11} \mathrm{~cm}^{-3}$ & $6 \times 10^{11} \mathrm{~cm}^{-3}$ & $1 \times 10^{8} \mathrm{~cm}^{-3}$ & $1.6 \times 10^{10} \mathrm{~cm}^{-3}$ & $10^{10} \mathrm{~cm}^{-3}$ & $10^{10} \mathrm{~cm}^{-3}$ \\
\hline
\end{tabular}




\section{Parametric optimization of mercury free signs}

\subsection{Pulse duration analysis}

As discussed above from the analysis of figure 2 , the second phase of the glow is dominated by the production of xenon ion, neon excited states and the successive increase of current amplitude and production of xenon ion excited states. The optimization of the post discharge fluorescence resulting from an increase of the xenon ion density during the glow phase could thus be experienced by the matching of the pulse duration. The figure 5 presents the temporal evolution of the current waveform, the resonant transition at $146.9 \mathrm{~nm}$ and of the xenon ion transition at $529.2 \mathrm{~nm}$ obtained in a 45 mbar $\mathrm{Ne} / \mathrm{Xe}(100 / 1)$ discharge excited respectively by two and five microsecond duration pulses.
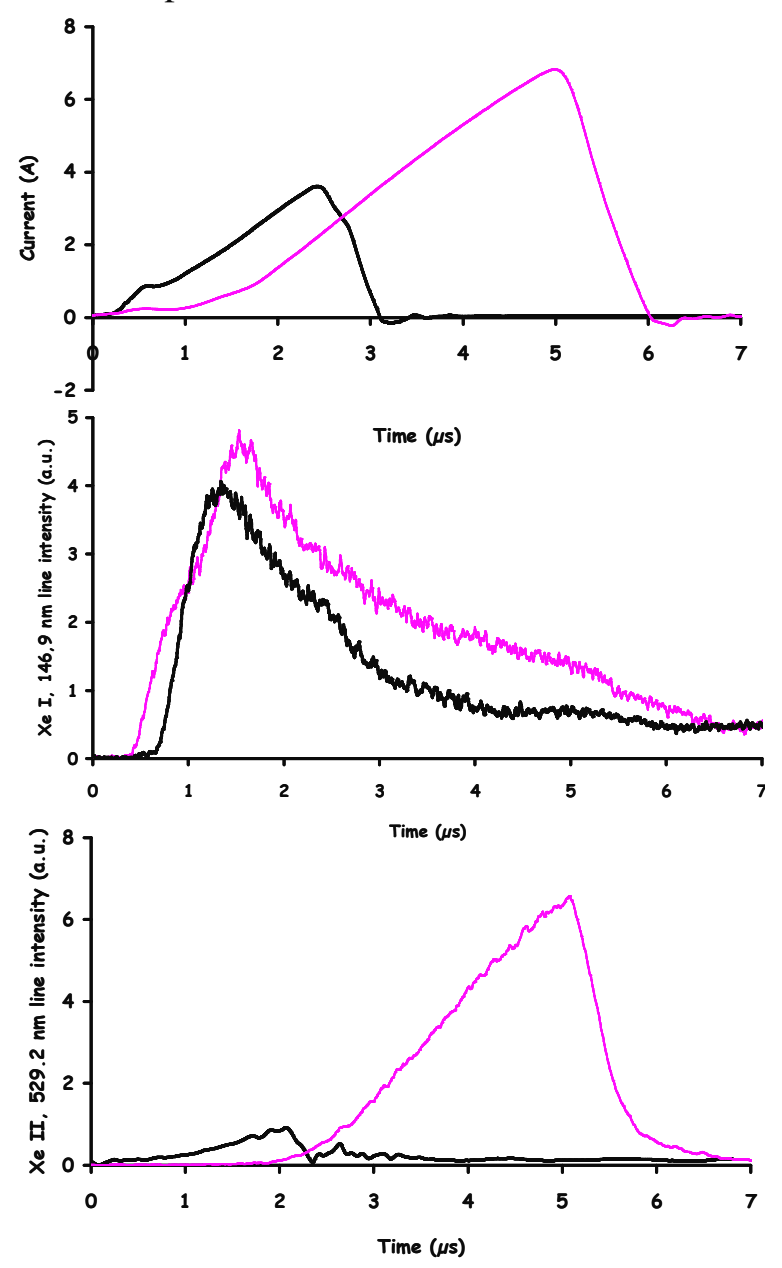

Figure 5. Current across a 45 mbar Ne/Xe (100/1) tube, XeI $146.9 \mathrm{~nm}$ and XeII $529.2 \mathrm{~nm}$ waveforms for 2 microsecond (black traces) and 5 microsecond duration (grey traces) excitation pulses. In both cases, the electrical input power is set to $30 \mathrm{~W}$.
A linear growth of the plasma charge, obtained by the time integration of the current pulse over the glow period, was measured as a function of the pulse duration on the whole 1 to $20 \mu$ s domain. The figure 5 indicates that while no strong increase of the resonant radiation is observed for longer duration pulses, the enhancement of the population of the xenon ions is very significant, in agreement with the two phase analysis of the glow signal in figure 2. A consecutive increase of the VUV afterglow was obtained resulting from the recombination of a larger amount of molecular ions. The figure 6 presents the experimental $146.9 \mathrm{~nm}$ afterglow signal together with the results from the model for 2,5 and $10 \mu \mathrm{s}$ pulses. The modelling was performed using the reaction and rate sets of table 1 and the initial densities reported in table 2 . The initial electron temperature was kept to a constant value of $0.7 \mathrm{eV}$ with the same temporal evolution as depicted in figure 4 . The initial excited neon atom density was also kept constant, while it was experimentally observed that the neon transition intensity is enhanced through pulse duration increasing but this value has no critical impact on the simulated temporal profiles.

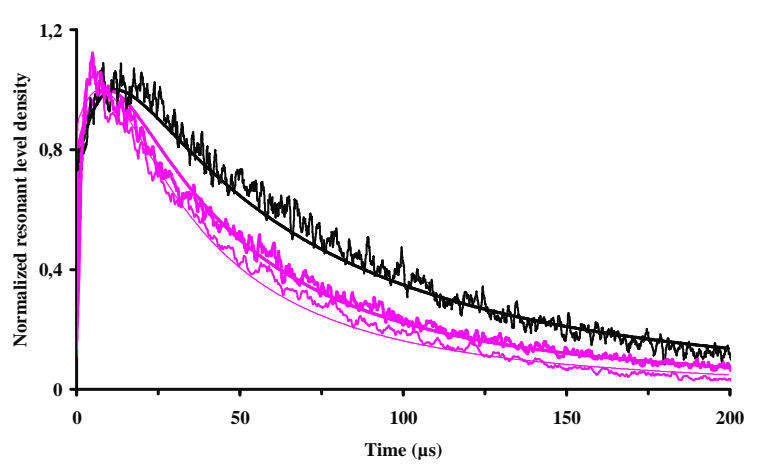

Figure 6. Experimental and simulated afterglows for 2 (black trace), 5 (thick grey trace) and $10 \mu \mathrm{s}$ (thin grey trace) pulses. The calculations use reactions and rates from table 1 and initial densities from table 2 .

Table 2. Species initial densities for figure 6 calculations.

\begin{tabular}{|c|c|c|c|}
\hline $\begin{array}{c}\text { Pulse } \\
\text { duration } \\
(\mu \mathrm{s})\end{array}$ & $\mathrm{e}^{-} / \mathrm{Xe}^{+}$ & $\mathrm{Xe}^{*}$ & $\mathrm{Xe}_{2}{ }^{+} / \mathrm{NeXe}^{+}$ \\
\hline 2 & $610^{11}$ & $1.610^{10}$ & $10^{10}$ \\
\hline 5 & $1.110^{12}$ & $210^{10}$ & $510^{10}$ \\
\hline 10 & $1.510^{12}$ & $210^{10}$ & $1.110^{11}$ \\
\hline
\end{tabular}


It appears from figure 6 that the rough modelling of the afterglow kinetics is consistent with the experimental data if modulation of the initial density values is performed in agreement with the behaviour measured for resonant and xenon ion transitions such as illustrated in figure 5 .

While confirming the simplified description of the plasma kinetics in neon xenon mixtures, the increase of the pulse duration must be counterbalanced by an appropriate lowering of the pulse repetition rate to obtain a constant electrical input power which is of crucial importance for the efficiency issue of any light source. The figure 7 presents the evolution of the illuminance as a function of the pulse duration in a 45 mbar neon-xenon (100-1) mixture for two lab developed pulsed drivers.

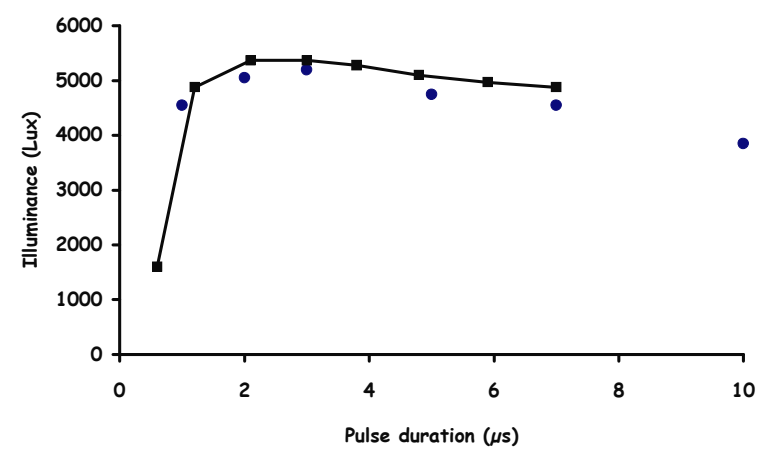

Figure 7. Evolution of the illuminance versus the pulse duration for a constant power across the sign with driver 1 (dots) and for a constant wall plug power input with driver 2 (square fitted trace). $\mathrm{Ne} / \mathrm{Xe}(100 / 1)$ mixture at a pressure of 45 mbar.

The data measured with the pulsed driver 1 including a voltage transformer to deliver high voltage pulse with an amplitude in the kilovolt range as described in the experimental set up section, have been obtained by keeping a constant electrical input across the sign regardless of the wall plug power which increases together with the pulse duration due to the power dissipation in the leak inductance of the transformer. A new pulsed driver 2 was designed with a $\mathrm{H}$ bridge inverter operating at voltage as high as $1500 \mathrm{~V}$ which allow both to suppress the power dissipation in the output transformer and to apply pulse with duration as low as 0,35 microseconds. In this latter case, the full line data in figure 7 corresponds to the evolution of the illuminance with the pulse duration for a constant electrical input of $30 \mathrm{~W}$ across the sign and also a constant wall plug power of about $35 \mathrm{~W}$. It appears from figure 7 that there exists an optimum for the phosphor excitation efficiency for pulse duration ranging from two to five microseconds. For larger pulse duration, the high peak current amplitude leads to a small contraction of the positive column plasma along its longitudinal axis which results in a smaller VUV radiating plasma surface and thus a decrease of the tube illuminance. On the other hand, the reduction of the pulse duration below two microseconds correspond to the production of lower xenon ion and neon excited states densities which results in a very reduced afterglow fluorescence contribution and a smaller sign efficiency even at high pulse repetition rate. This last result indicates that in our experimental conditions, the best performances are achieved with an efficient recovery of the energy spent during the glow phase involving stepwise ionisation and ionization processes. For shortest pulse duration operation, the limitation of these a priori undesirable processes is nevertheless less favourable for the sign efficiency.

\subsection{Role of the pressure}

The other parameter very likely to have a significant effect on the afterglow optimization is the mixture gas pressure. Both the xenon and neon pressure increase could play a positive role in the production of higher molecular ions $\left(\mathrm{Xe}_{2}{ }^{+}\right.$or $\left.\mathrm{NeXe}^{+}\right)$densities. The increase of the xenon partial pressure was shown to be possible over a very limited range below a few mbars. As the xenon pressure is increased the discharge first contracts along the longitudinal axis and rapidly turns from a diffuse glow mode to a filamentary or arc mode which is non stationary, inducing premature damage to the electrodes and not allowing an efficient excitation of the phosphor layer. These limitations are probably strongly connected with the about three orders of magnitude lower secondary emission coefficient of xenon ions in comparison with that of neon ions [19]. As argued in section 3, in our experimental conditions, the pulsed excitation of neon xenon with small admixtures of xenon of a few per cent, does not lead to the efficient population of the excited dimer levels radiating the first and second continua on the red wing of the resonant transition. The figure 8 presents the 
VUV spectrum of a 100 mbar $\mathrm{Ne} / \mathrm{Xe}(100 / 1)$ discharge powered by the pulse driver for an electrical input of $30 \mathrm{~W}$, which shows no indication for the xenon dimer radiation. The main emission occurs at $146.9 \mathrm{~nm}$, and the $129.6 \mathrm{~nm}$ and $119.2 \mathrm{~nm}$ resonant radiations are also detectable but with much more lower intensity. On the contrary, previous higher resolution VUV measurements [6] have shown that the $(\mathrm{NeXe})^{*}$ exciplex transitions are observed on the blue wing of the xenon 146.9 resonant line. In this later case, termolecular reactions inducing one xenon atom with two neon atoms probably account for the population of the upper state of the $(\mathrm{NeXe})^{*}$ exciplex which in our experimental conditions, i.e. high neon density, is a much more probable reaction that those required for the xenon dimer formation for comparable reaction rate values. The influence of such termolecular reaction for $(\mathrm{NeXe})^{*}$ population was nevertheless not included in the modelling calculation.

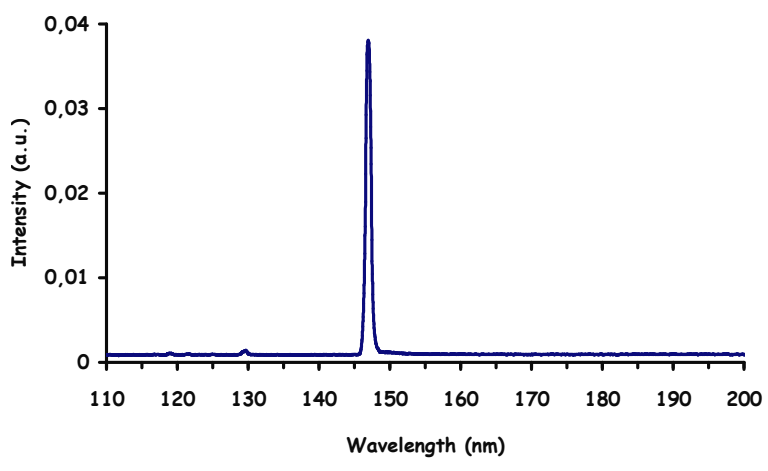

Figure 8. VUV spectrum of a 100 mbar $\mathrm{Ne} / \mathrm{Xe}$ (100/1) discharge using pulsed excitation and an electrical input power of $30 \mathrm{~W}$. A vertical offset was applied for clarity of the figure. The spectrum is not corrected for the spectrometer sensitivity over the VUV spectral range.

The illuminance evolution with the pressure for mixtures containing $1 \%$ of xenon is presented in figure 9 . The data in figure 9 were obtained by using 2 microsecond duration pulses and a constant input power across the sign of $20 \mathrm{~W}$, this latter condition was achieved by a slight increase of the pulse repetition rate over the pressure range. The best sign efficiency is obtained for mixture pressures between 60 to 80 mbar. The progressive reduction of the increase of illuminance level for each 10 mbar increment up to its saturation around 70 mbar and the slight decrease of the illuminance for the higher pressures are observed. This phosphor behaviour is quite different of that of the VUV intensity evolution measured through the vacuum evacuated glass capillary in the phosphor plane also presented in figure 9. The deviation between the two measurements originates from the progressive contraction of the positive column as the mixture pressure is increased.

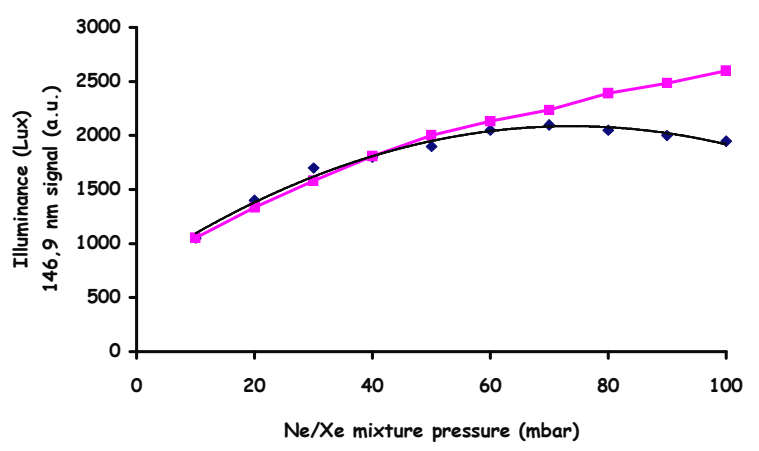

Figure 9. Evolution of the illuminance versus the pressure (lozenge dark fitted trace) and evolution of the $146.9 \mathrm{~nm}$ intensity with pressure (square grey fitted trace)

The glass capillary visualized a constant surface of the plasma column which is optically thick for the resonance line radiation, the surface of the magnesium fluoride window being always smaller in our experimental conditions than the plasma column surface even for the largest pressures. The plasma column diameter was measured through a visible ICCD imaging experiment and it evolves from $13 \mathrm{~mm}$ at 10 mbar down to $9 \mathrm{~mm}$ at $100 \mathrm{mbar}$ for a $20 \mathrm{~W}$ tube input power. With the assumption that the VUV radiation radial distribution is not considerably different to that of the visible part of the spectrum, it appears that the VUV capillary measurement is not influenced by the contraction of the plasma column while the phosphor is excited by a smaller and smaller VUV emitting surface as the pressure is raised.

In comparison with the case of pure xenon plasma, the contraction is observed while the plasma column is homogeneous and not filamentary up to $100 \mathrm{mbar}$ if the power applied across the sign is higher than about 10 W. It was also measured that the contraction appears for higher pressures as the tube diameter is reduced in agreement with some 
previous analysis $[20,21]$ and that the contraction could be counterbalanced by an increase of the power injected across the sign. It was finally measured [6] that besides the $146.9 \mathrm{~nm}$ resonant radiation, part of the phosphor excitation originates from the $(\mathrm{NeXe})^{*}$ excimer, radiated in the blue wing of the xenon resonant line, as the mixture pressure is increased. This excimer contribution was estimated to be of about $10 \%$ at 50 mbar and up to $15 \%$ at 90 mbar of the whole VUV radiated intensity, and is not trapped during its propagation from the plasma to the phosphor layer, at the difference of the resonance line. These observations and the consideration that the VUV intensity is increasing in a small volume around the tube axis, as depicted by VUV data in figure 9, may be of interest for the development of efficient higher pressure and small diameter mercury free signs.

\section{Lifetime and efficiency studies}

The results of this section were obtained in sealed signs and thus include the potential cataphoresis, and any other long term plasma phosphor, glass and electrode interaction effects. With AC excitation, the continuous operation of neon xenon signs results in a selective trapping of xenon in the glass tube and tube electrodes which induces strong limitation on the lifetime of the signs [10, 22]. For AC excitation, there exists a strong trade off between the lifetime and the visible output of the signs. On one hand, AC driven neon xenon signs offering the greatest illuminance level, which was measured to be lower than 1000 Lux for the green phosphor used in this work, unfortunately presents lifetime of a few tens of hours. On the other hand, successful continuous operation during time periods of 10 to 12 thousands of hours were measured but with illuminance output of a few hundreds of Lux. In both cases, AC driven mercury free systems exhibit performances which do not allow their development for publicity lightning applications. In the case of pulsed excitation, the optimization of neon xenon plasmas was obtained for mixture pressures of a few tens of mbar and ageing studies have shown successful operation of mercury free signs delivering illuminance ranging from 2000 to 3000 Lux during time periods of at least 4000 hours. The continuous operation of a $50 \mathrm{mbar}$, $1 \%$ neon xenon signs also indicate that the xenon trapping is considerably reduced as the chromatic coordinates ( $\mathrm{x}$ and $\mathrm{y}$ following the CIE definition) exhibits a constant value during the 4000 hours experiment. As an illustration, the figure 10 presents the ageing study performed for a 50 mbar $\mathrm{Ne} / \mathrm{Xe}(100 / 5)$ sealed sign continuously powered at $30 \mathrm{~W}$. The illuminance and chromatic coordinates both measured with the luxmeter were recorded during 2800 hours with a mean sampling interval of 50 hours. The $\mathrm{x}$ and $\mathrm{y}$ chromatic coordinates for mercury based low pressure mixtures using the same green phosphor are respectively of 0.25 and 0.59 , which are very close to that obtained with neon xenon mixtures having a sufficient content of xenon to efficiently quench the neon red transition radiations.

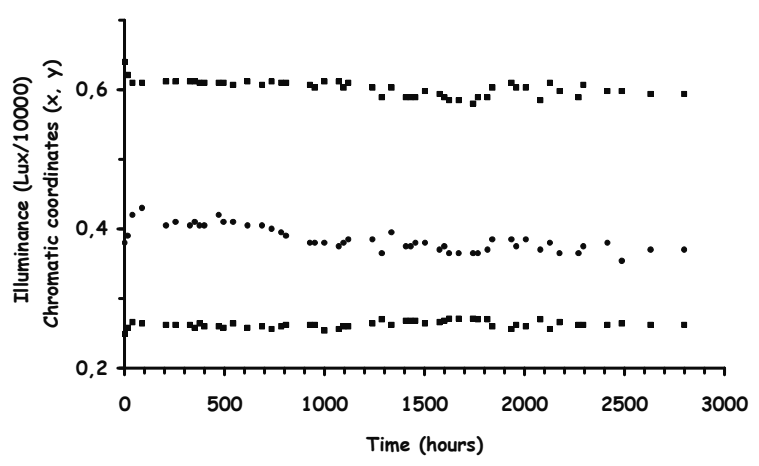

Figure 10. Evolution of chromatic coordinates (squares, $\mathrm{x}$ mean 0.26, y mean 0.60) and illuminance (dots, mean value 3850) for a $50 \mathrm{mbar}$ $\mathrm{Ne} / \mathrm{Xe}(100 / 5)$ sign.

The measurements were stopped after 2800 hours. No sputtering of the electrodes was observed as revealed both by the electrode outer surface aspect which show no evidence of demetallization together with the absence of any deposition layer on the inside surface of the sign in the vicinity of the electrodes which was the first indication of the electrode degradation in AC driven low pressure tubes. 


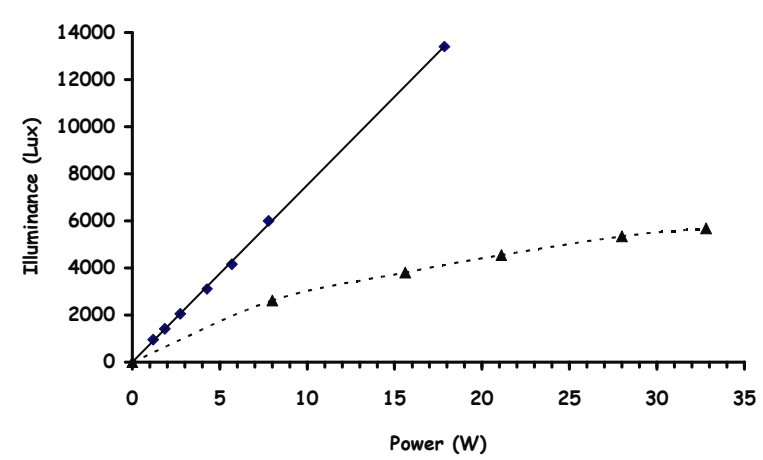

Figure 11. Evolution of the illuminance with the input power for mercury based sign (lozenge fitted trace) and for neon xenon sign (triangle dashed fitted trace).

The figure 11 compares the evolution of the illuminance for $13 \mathrm{~mm}$ inside diameter, $50 \mathrm{~cm}$ long fluorescent tube as a function of the electrical power input for a $\mathrm{Ar} / \mathrm{Ne} / \mathrm{Hg} 10 \mathrm{mbar}$ and a $\mathrm{Ne} / \mathrm{Xe}(100 / 1) 60$ mbar sealed signs. In both cases, the inside surface of the sign is covered by a green emitting phosphor layer processed by the same industrial procedure. While the mercury based sign exhibits a constant slope of the illuminance increasing versus the electrical power, the $\mathrm{Ne} / \mathrm{Xe}$ sign illuminance tends to gradually saturate to a value of about 6000 Lux for an electrical input around $40 \mathrm{~W}$. It must be reminded that a significant improvement of the mercury free signs performance was obtained first through the use of pulsed excitation in comparison with the $\mathrm{AC}$ excitation, in this latter the illuminance value never exceeds 1000 Lux whatever the electrical input is, and also thanks to the optimization of the filling pressure and pulse duration. These features also hold true for longer signs experienced up to $2 \mathrm{~m}$ in length and smaller $(11 \mathrm{~mm})$ or larger $(18 \mathrm{~mm})$ inside diameter tubes.

Complementary experiments were performed with the use of the integrating sphere. These measurements indicate first that the efficiency of the mercury based green signs is almost constant over the power range studied in this work up to $50 \mathrm{~W}$, in agreement with the illuminance behaviour depicted in figure 10 , and has a value of $30 \mathrm{Lm} / \mathrm{W}$ for $13 \mathrm{~mm}$ inside diameter tubes. The figure 12 presents the efficiency of four different mercury free $50 \mathrm{~cm}$ long signs, with one per cent of xenon content, as a function of the electrical power. The decrease of the efficiency is almost identical for all the signs for injected power higher than $15 \mathrm{~W}$, whatever the pressure $(45,60$ or 80 mbar) and the tube diameter (13 or $18 \mathrm{~mm}$ ) for the 80 mbar filling. For electrical power lower than $15 \mathrm{~W}$, the slightly higher efficiency of 13 $\mathrm{mm}$ diameter in comparison with the $18 \mathrm{~mm}$ illustrated for the pressure of $80 \mathrm{mbar}$ is representative of the behaviour of $\mathrm{Ne} / \mathrm{Xe}$ signs all over the pressure range studied in this work from 10 mbar up to 100 mbar. As depicted for the $13 \mathrm{~mm}$ diameter tubes, there exists an optimum for the sign efficiency which appears with larger and larger amplitude and for lower and lower electrical power when the tube pressure is decreased. For the $\mathbf{4 5}$ mbar pressure this efficiency maximum is not measured and is probably achieved for very low electrical power, below $2 \mathrm{~W}$.

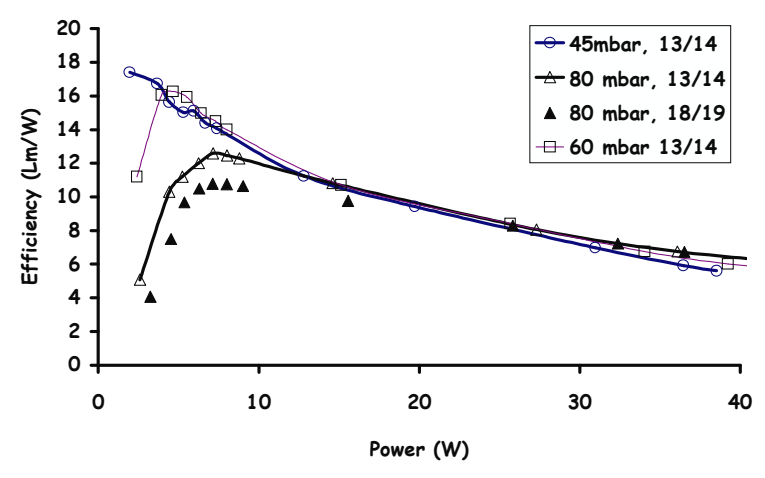

Figure 12. Evolution of the efficiency with the input power for different mercury free signs which pressure and inner/outer diameter are labelled in the inset. The xenon content is always of one per cent. The efficiency of mercury based signs of the same geometry and same phosphor coating is measured to be of about $30 \mathrm{Lm} / \mathrm{W}$.

For power input smaller than $15 \mathrm{~W}$, the reduction of the efficiency with the pressure increase is attributed to the energy consumption in the electrode region of the discharge. Thus for a given electrical input power, a larger proportion of it is dissipated for the discharge maintenance considering that the pressure increase has a quite insignificant effect on the secondary emission coefficient [19] while it has a strong effect on the breakdown voltage for pure neon and even more drastically for neon -xenon mixtures. 


\section{Conclusion}

It follows from the analysis of the pressure, the efficiency and the lifetime performance studies that medium pressure, i.e. around 50 mbar, neon xenon mixtures containing a few amount, around $1 \%$, of xenon provide green light output of a few hundreds of lumen with an efficiency of about $50 \%$ of that of mercury based signs and lifetime of a few thousands of hours. These features appear of interest for the development of mercury free interior signs and may also be valuable for rather low visible output applications requiring low injected electric power which correspond to higher efficiency operation of neon xenon plasma. It must be noticed that the mercury free sign performances are obtained without any change neither in the electrode arrangement nor in the green phosphor chemical composition, which allow for a very easy manufacturing considering that only the mercury and gas mixture blend as to be changed for a neon xenon filling. On the other hand, the design of specific phosphor likely to be much more efficient under $147 \mathrm{~nm}$ excitation is a very motivating issue to achieve substantial enhancement of the sign efficiency. The use of external electrodes eventually in combination with the conventional cold hollowed electrodes may also be of interest for the production of homogeneous higher pressure plasma [23].

\section{Acknowledgments}

This work is supported by AUPEM SEFLI company, the French Agency for Environment and Energy Management (ADEME) and la Région Centre. The authors are grateful to J. Mathias for VUV measurements and M. Pretre, J. Duchene and G. Cailleaux for their contribution to the experimental parametric study during their training periods.

\section{References}

[1] G. Zissis, Proc. of the $10^{\text {th }}$ Int. Symp. on the Science and Technology of Light Sources, IOP Conf. Series Number 182 (2004)

[2] M.Q. Liu and R. Devonshire, Proc. of the $11^{\text {th }}$ Int. Symp. on the Science and Technology of Light Sources, FAST-LS (2007)

[3] J. Phys. D: Appl. Phys., special issue, 38 (2005)
[4] D. Uhrlandt, R. Bussiahn, S. Gorchakov, H. Lange, D. Loffhagen and D. Nötzold, J. Phys. D: Appl. Phys., 38, 3318-3325 (2005)

[5] Yu B. Golubovskii, I.A. Porokhova, H. Lange and D. Uhrlandt, Plasma Sources Sci. Technol., 14, 36-44 (2005)

[6] S. Point, E. Robert, S. Dozias, C. Cachoncinlle, R. Viladrosa and J.M. Pouvesle, Journal of Optoelectronics and Advanced Materials, 10, 19221926 (2008)

[7] S. Gortchakov and D. Uhrlandt, Plasma Sources Sci. Technol., 14, 177-183 (2005)

[8] Yu B. Golubovskii, I.A. Porokhova, H. Lange, S. Gortchakov and D. Uhrlandt, Plasma Sources Sci. Technol., 14, 45-50 (2005)

[9] T. J. Sommerer, J. Phys. D: Appl. Phys., 29, 769-778 (1996)

[10] E. Robert, H. Sarroukh, C. Cachoncinlle, R. Viladrosa, V. Hochet, S. Eddaoui and J.M. Pouvesle, Pure Appl. Chem., 77 (2), 463-474 (2005)

[11] S. Point, E. Robert, S. Dozias, C. Cachoncinlle, R. Viladrosa and J.M. Pouvesle, J. Phys. IV, 138, 105-110 (2006)

[12] J. B. Boffard, R.O. Jung, L.W. Anderson and Chun C. Lin, Adv. Atom. Mol. Opt. Phys., 54, 319421 (2006)

[13] R.H. Neynaber and S.Y. Tang, J. Chem. Phys., 70, 4272-4276 (1979)

[14] D.J. Eckstrom, H.H. Nakano, D.C. Lorents and T. Rothem, J. Appl. Phys., 64, 1679-1690 (1988)

[15] L.A. Levin, IEEE J. Quant. Electr., 17, 2282 (1985)

[16] J. Meunier, Ph. Belenguer and J.P. Bœuf, J. Appl. Phys., 78, 731-745 (1995)

[17] Yu B. Golubovskii, H. Lange, V.A. Maiorov, I.A. Porokova and V.P. Sushkov, J. Phys. D: Appl. Phys., 36, 694-703 (2003)

[18] B. Min, S.H. Lee, H.G. Park, J. Vac. Sci. Technol. A, 18, 349 (2000)

[19] H. Capdeville, Ph. Guillot, L.C. Pitchford, J. Galy and H. Brunet, Proc. XXIV ICPIG, III, 5 (1999)

[20] Y.B. Baranov and K.N. Ul'yanov, Zh. Tekh. Fiz, 39, 249 (1969). Y.B. Baranov and K.N. Ul'yanov, Sov. Phys. -Tech. Phys., 14, 176 (1969)

[21] G. Petrov and C.M. Ferreira, Phys. Rev. E, 59, 3571 (1999)

[22] H. Stafast, L. Redlich and H-P. Linke, J. Phys. D: Appl. Phys., 39, 4611-4615 (2006)

[23] M. Jinno, M. Okamoto, M. Takeda and H. Motomura, J. Phys. D: Appl. Phys., 40, 3889-3895 (2007) 


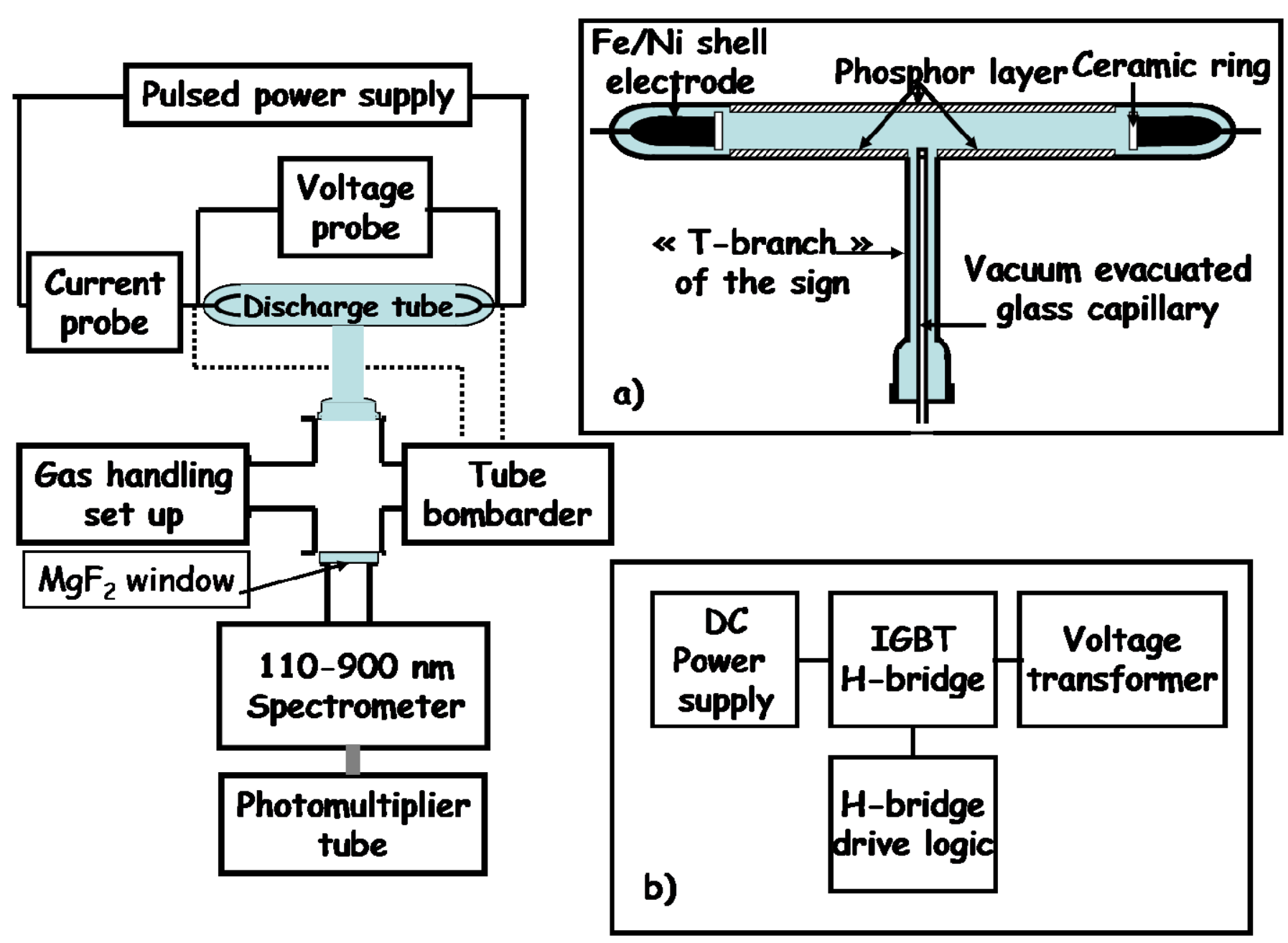

Figure 1 (fig1_ROBERT.tif) 

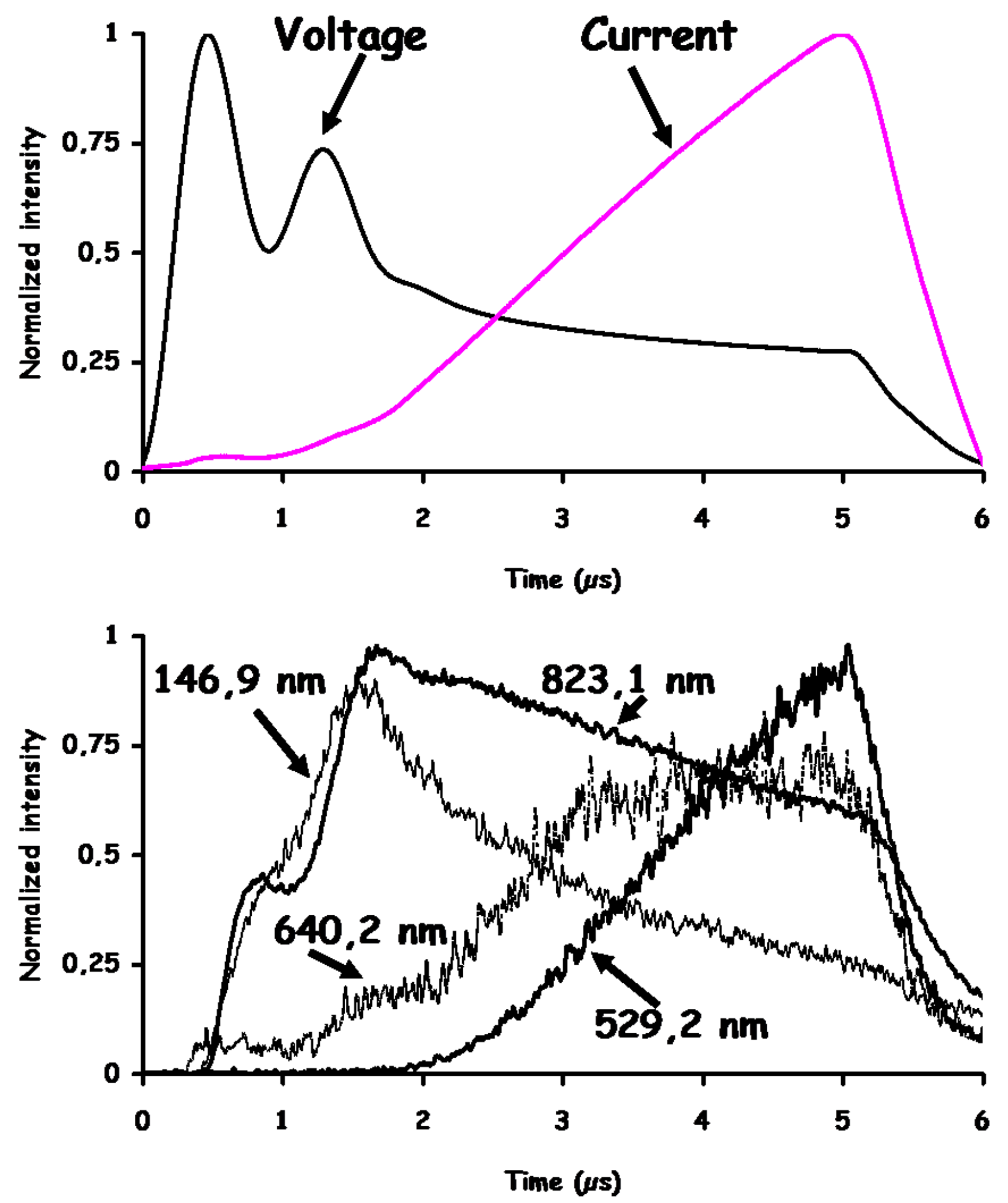

Figure 2 (fig2_ROBERT.tif) 


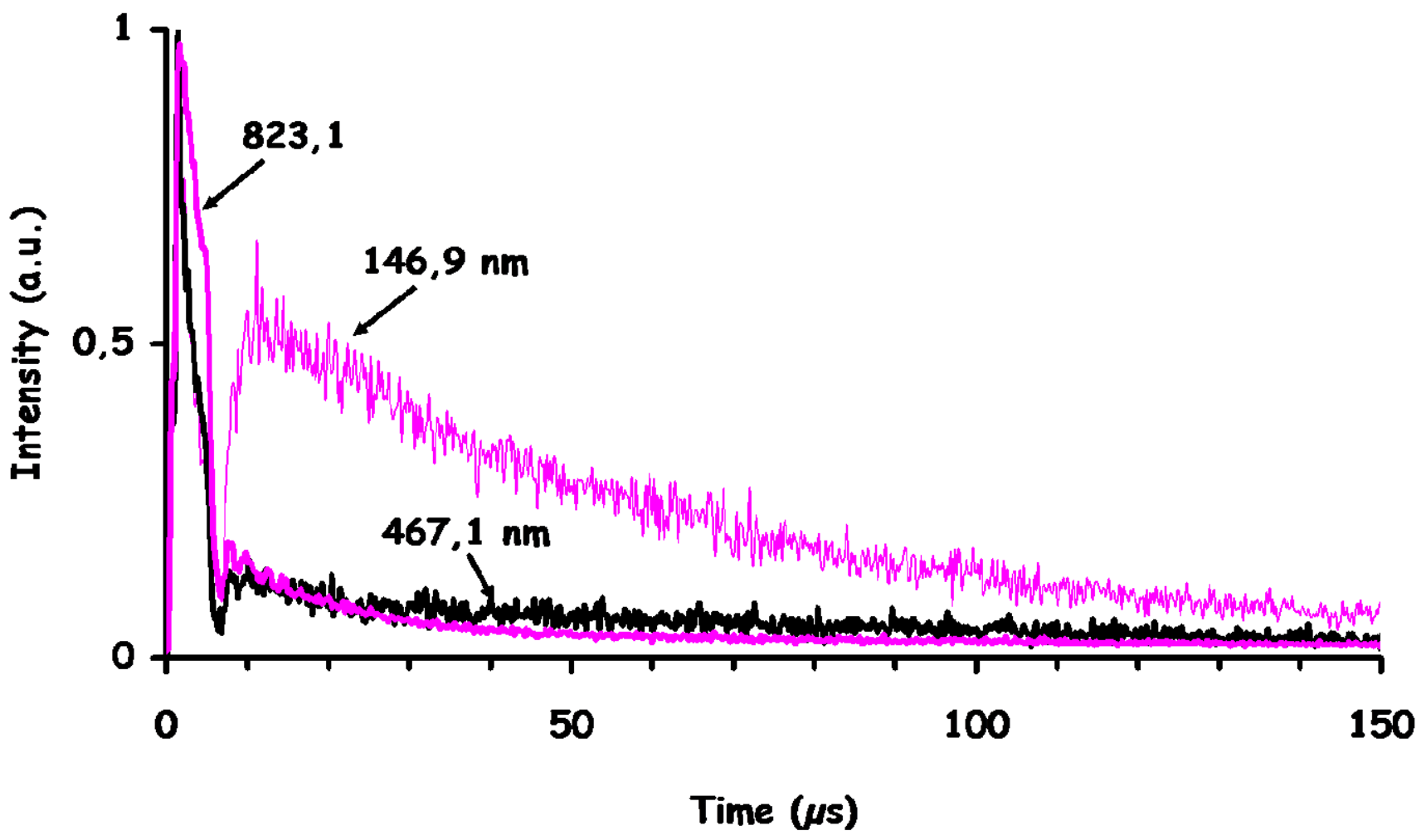

Figure 3 (fig3_ROBERT.tif) 


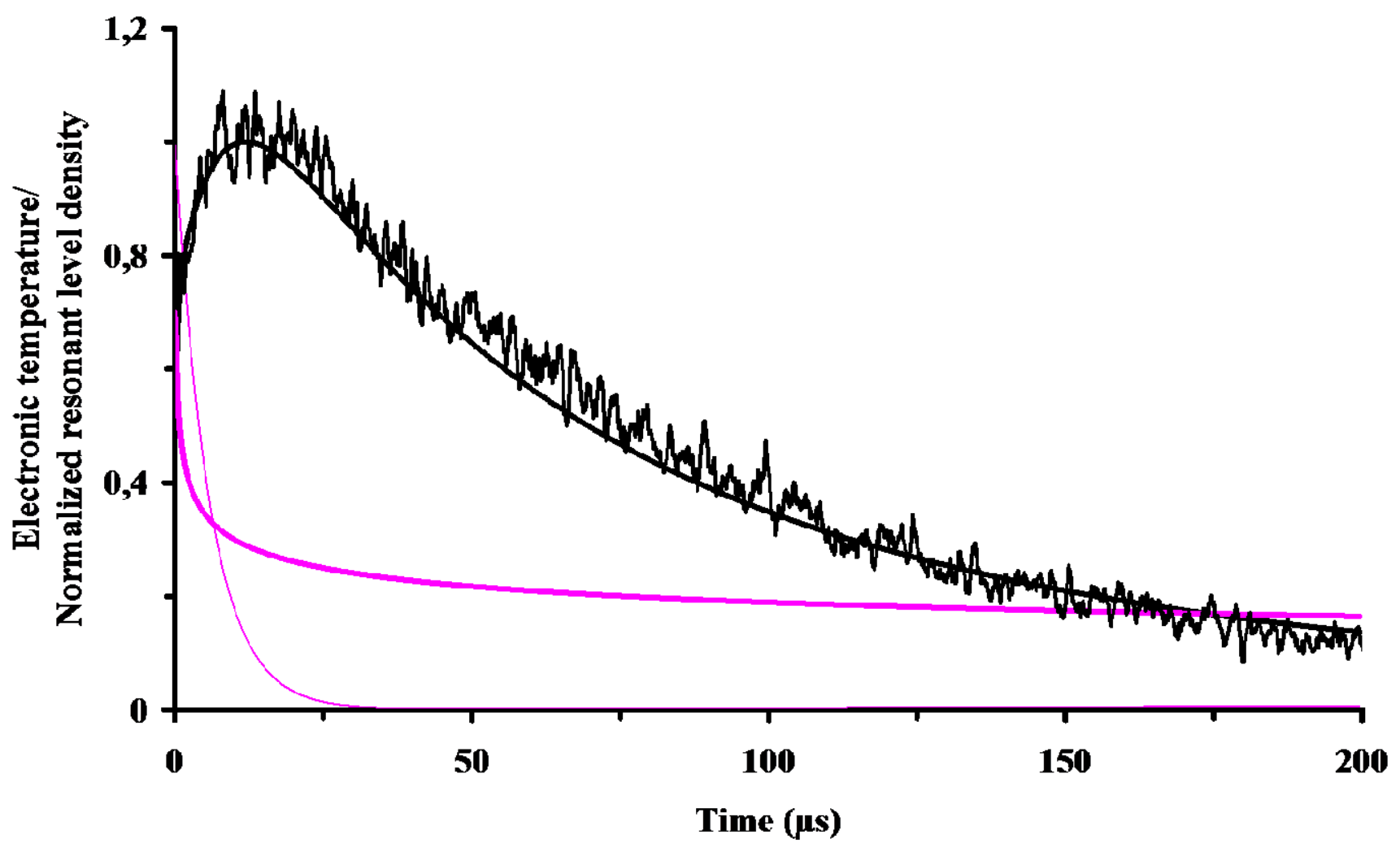

Figure 4 (fig4_ROBERT.tif) 


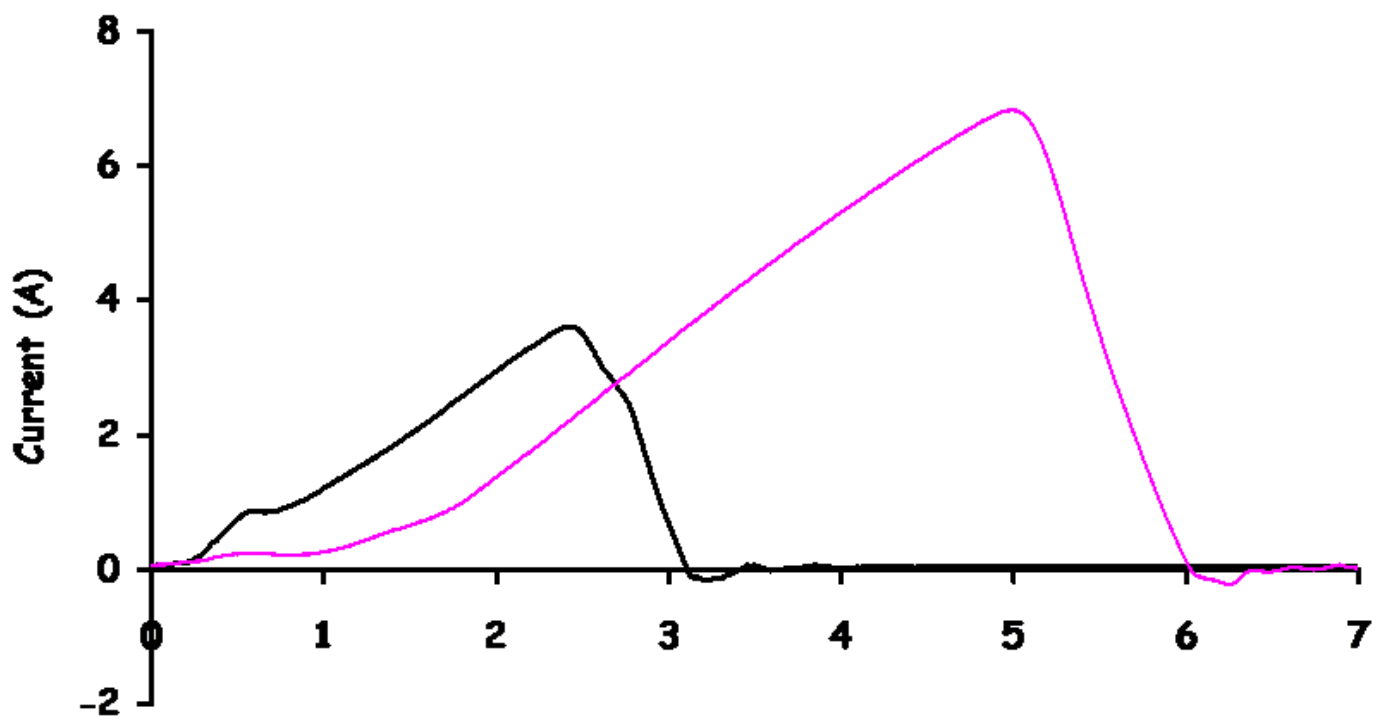

Time (us)
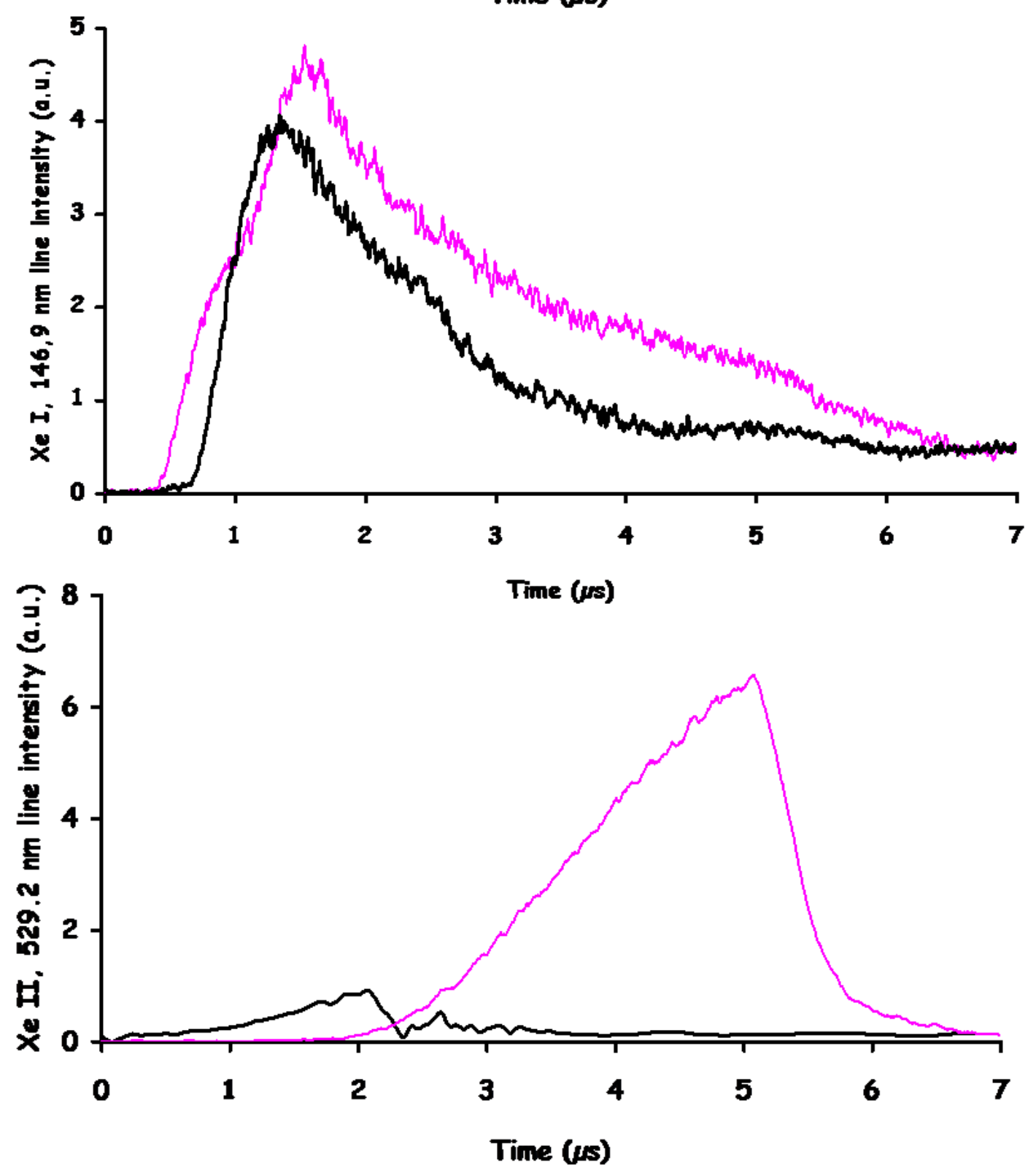

Figure 5 (fig5_robert.tif) 


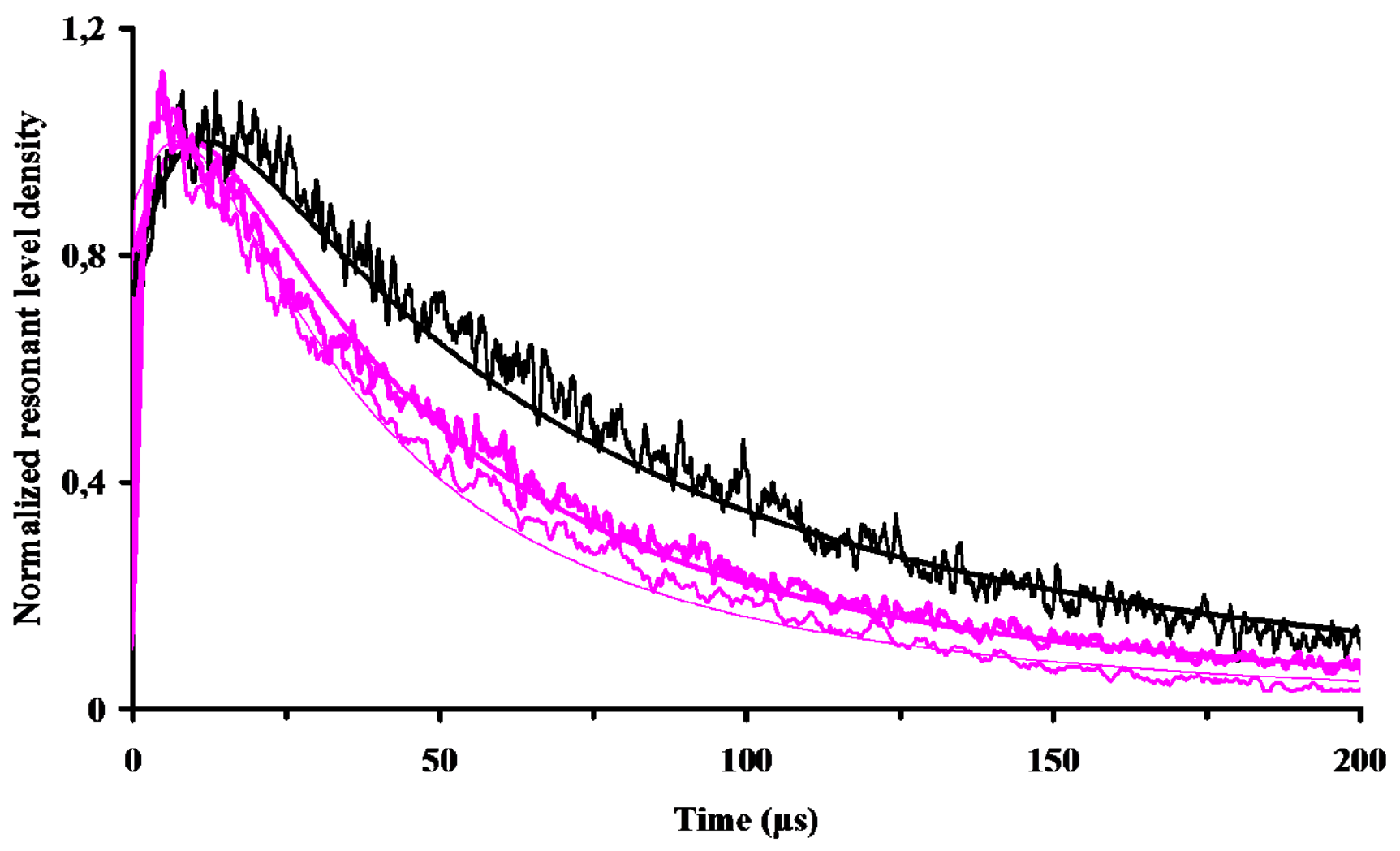

Figure 6 (fig6_ROBERT.tif) 


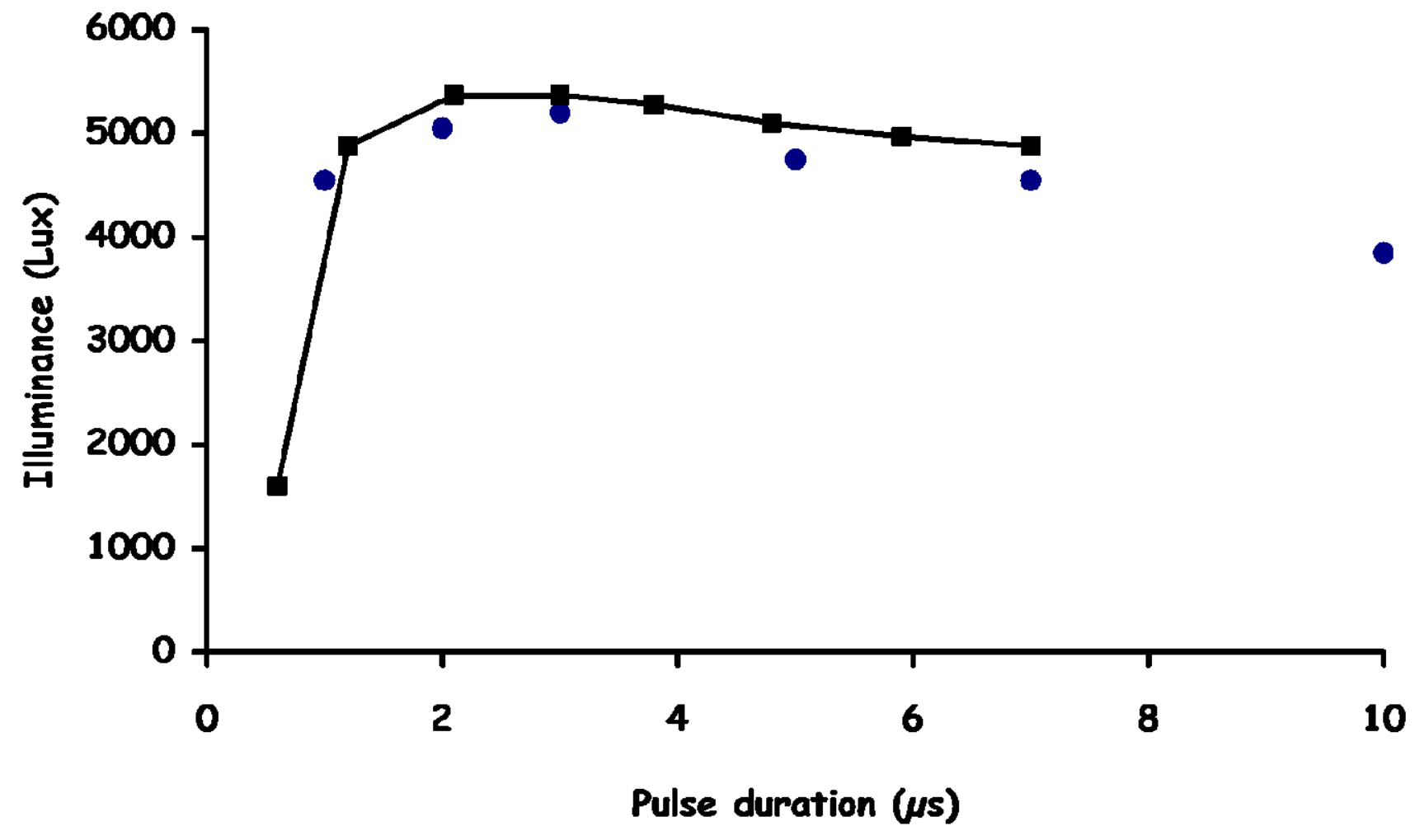

Figure 7 (fig7_ROBERT.tif) 


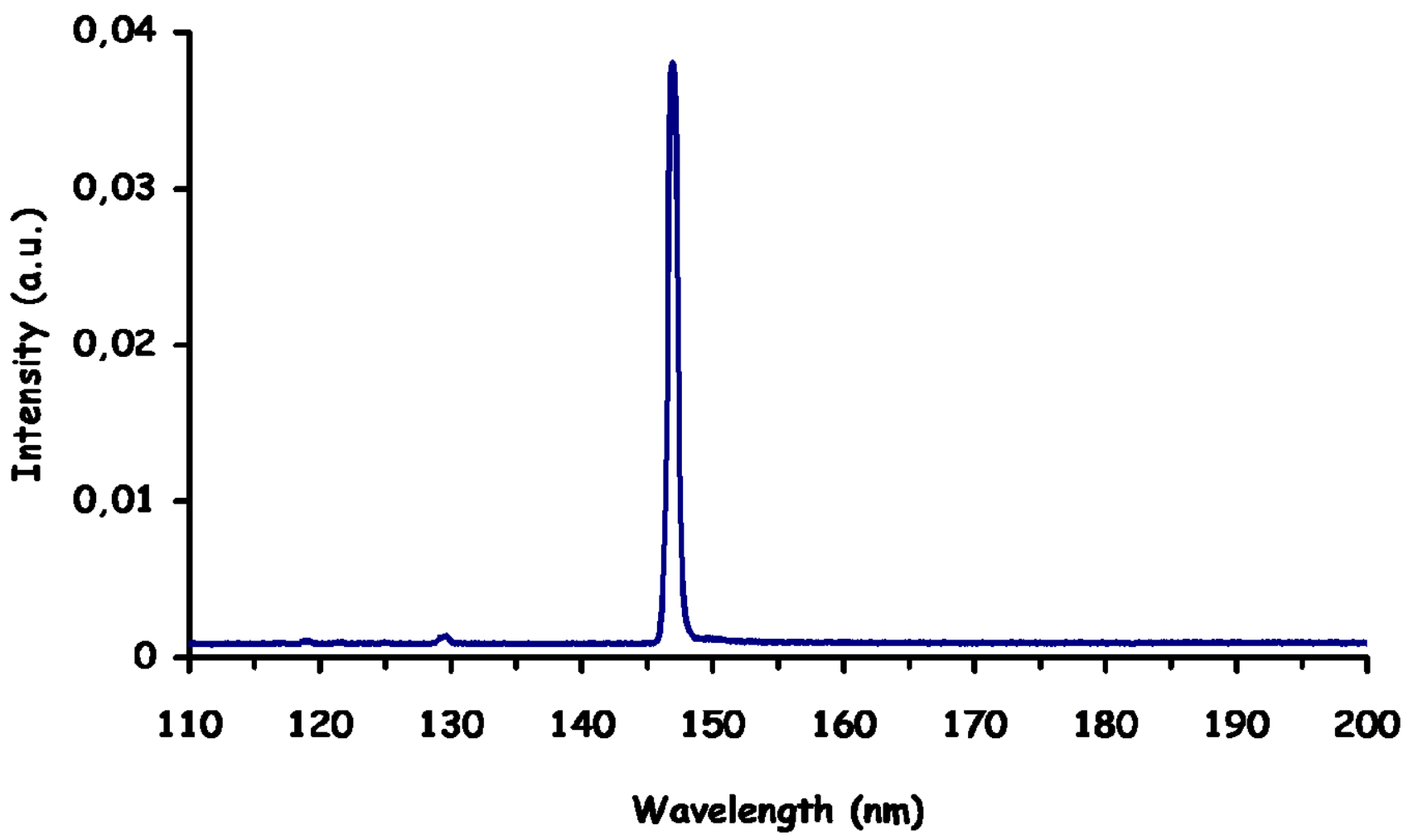

Figure 8 (fig8_ROBERT.tif) 


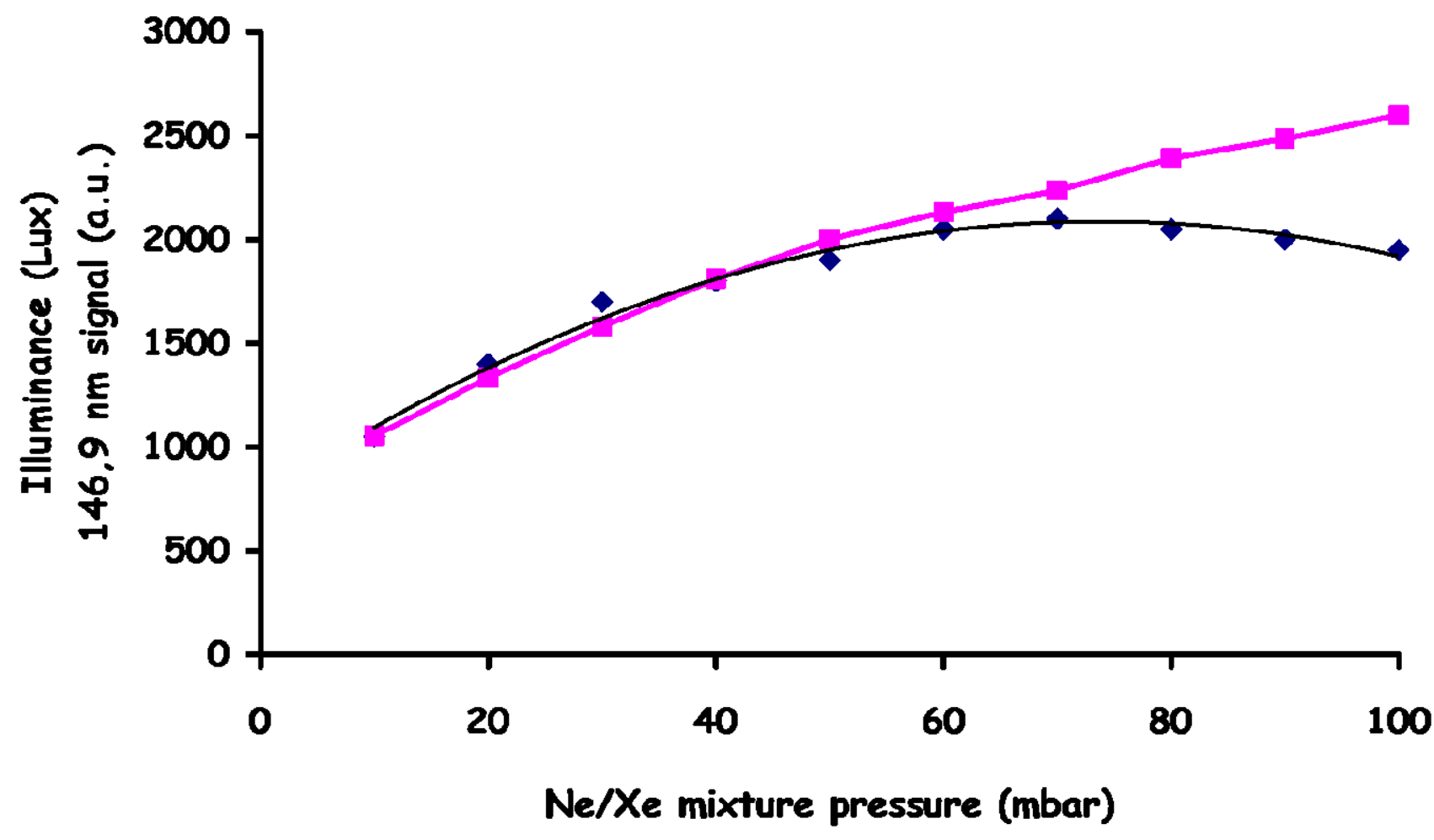

Figure 9 (fig9_ROBERT.tif) 


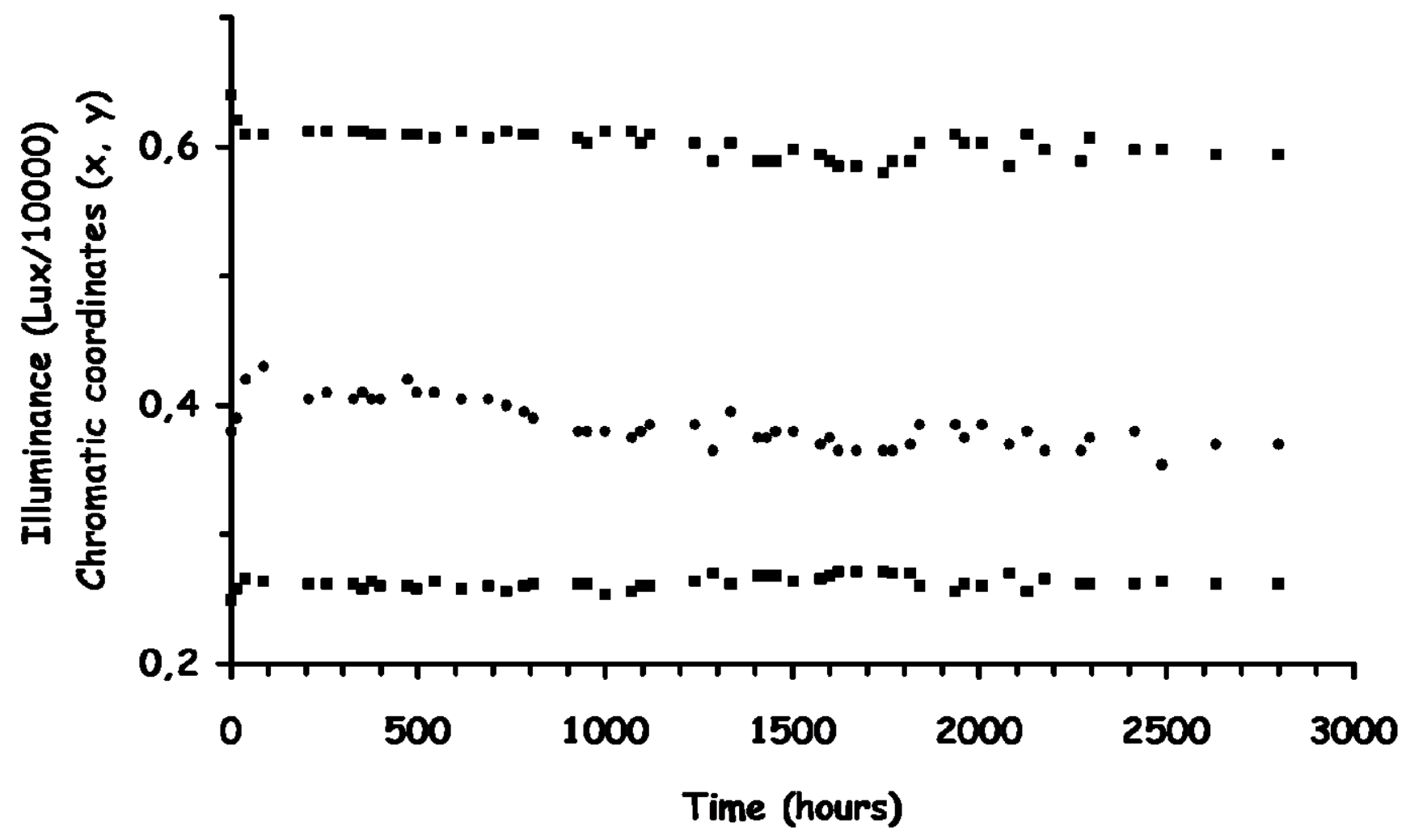

Figure 10 (fig10_ROBERT.tif) 


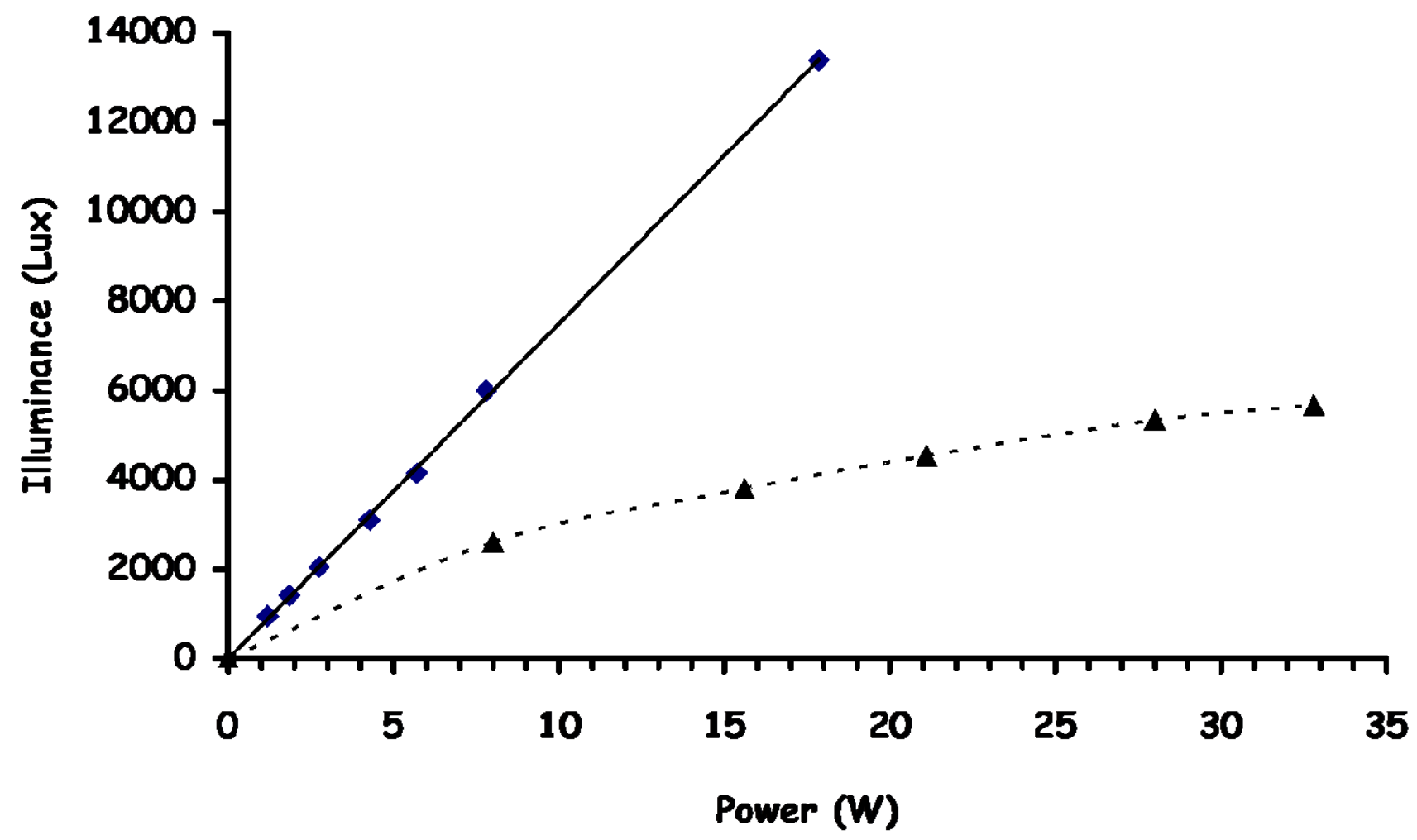

Figure 11 (fig11_ROBERT.tif) 


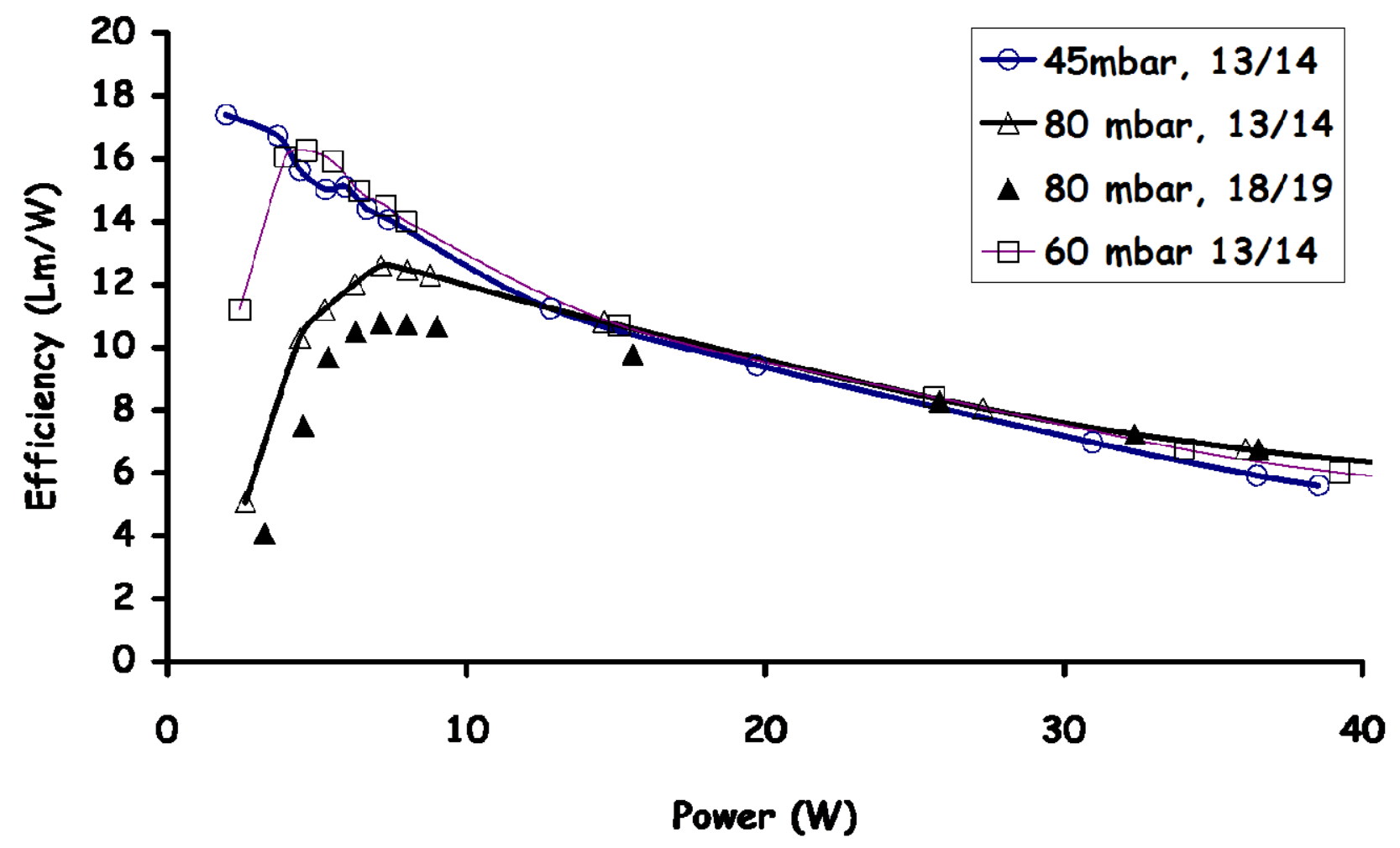

Figure 12 (fig12_ROBERT.tif) 